\title{
Molecular Beam Epitaxy of Layered Group III Metal Chalcogenides on GaAs(001) Substrates
}

\author{
Sergey V. Sorokin ${ }^{1, *}$, Pavel S. Avdienko ${ }^{1}$, Irina V. Sedova ${ }^{1}$, Demid A. Kirilenko ${ }^{1}$, \\ Valery Yu. Davydov ${ }^{1}{ }^{(}$, Oleg S. Komkov $\left.{ }^{2}{ }^{(}\right)$, Dmitrii D. Firsov ${ }^{2}$ and Sergey V. Ivanov ${ }^{1, *}$ \\ 1 Ioffe Institute, 26 Politekhnicheskaya, 194021 St. Petersburg, Russia; avdienko.pavel@gmail.com (P.S.A.); \\ irina@beam.ioffe.ru (I.V.S.); demid.kirilenko@mail.ioffe.ru (D.A.K.); valery.davydov@mail.ioffe.ru (V.Y.D.) \\ 2 St. Petersburg Electrotechnical University “LETI”, 5 Prof. Popova, 197376 St. Petersburg, Russia; \\ okomkov@yahoo.com (O.S.K.); d.d.firsov@gmail.com (D.D.F.) \\ * Correspondence: sorokin@beam.ioffe.ru (S.V.S.); ivan@beam.ioffe.ru (S.V.I.)
}

Received: 29 June 2020; Accepted: 30 July 2020; Published: 5 August 2020

\begin{abstract}
Development of molecular beam epitaxy (MBE) of two-dimensional (2D) layered materials is an inevitable step in realizing novel devices based on 2D materials and heterostructures. However, due to existence of numerous polytypes and occurrence of additional phases, the synthesis of 2D films remains a difficult task. This paper reports on MBE growth of GaSe, InSe, and GaTe layers and related heterostructures on $\mathrm{GaAs}(001)$ substrates by using a Se valve cracking cell and group III metal effusion cells. The sophisticated self-consistent analysis of X-ray diffraction, transmission electron microscopy, and Raman spectroscopy data was used to establish the correlation between growth conditions, formed polytypes and additional phases, surface morphology and crystalline structure of the III-VI 2D layers. The photoluminescence and Raman spectra of the grown films are discussed in detail to confirm or correct the structural findings. The requirement of a high growth temperature for the fabrication of optically active 2D layers was confirmed for all materials. However, this also facilitated the strong diffusion of group III metals in III-VI and III-VI/II-VI heterostructures. In particular, the strong In diffusion into the underlying ZnSe layers was observed in $\mathrm{ZnSe} / \mathrm{InSe} / \mathrm{ZnSe}$ quantum well structures, and the Ga diffusion into the top InSe layer grown at $\sim 450^{\circ} \mathrm{C}$ was confirmed by the Raman data in the InSe/GaSe heterostructures. The results on fabrication of the GaSe/GaTe quantum well structures are presented as well, although the choice of optimum growth temperatures to make them optically active is still a challenge.
\end{abstract}

Keywords: III-metal chalcogenides; 2D materials; GaSe; InSe; GaTe; quantum wells; molecular beam epitaxy; GaAs(001) substrate; photoluminescence; Raman spectroscopy; transmission electron microscopy; X-ray diffraction

\section{Introduction}

Layered two-dimensional (2D) group III metal chalcogenides (MCs) (GaSe, GaTe, InSe, and others) are still of strong interest for the development of novel high-performance semiconductor devices due to their unique electronic and optical properties [1-3]. In particular, these materials have attracted great attention for the fabrication of field-effect transistors [4], solar cells [5,6], high-efficient photodetectors, and gas sensing devices [7,8]. InSe has significant potential for using in thermoelectric applications [9]. In addition, GaSe and GaTe are the nonlinear optical crystals and can be used for terahertz (THz) wave generation $[10,11]$.

One of the main reasons for intensive studies of 2D layered materials is that they exhibit different properties in a few-layer and bulk forms [12-14], providing an additional degree of freedom in the development of electronic and optical devices. To date, most of the 2D layered semiconductors and 
heterostructures based on them are either prepared by mechanical exfoliation from bulk crystals or grown by chemical (physical) vapor transport (CVT, PVT) or chemical vapor deposition (CVD) techniques [15]. Although the sophisticated multilayered structures consisting of various 2D materials bonded to each other via weak van der Waals forces can be produced using the scotch-tape method ("realizing the idea that 2D materials can be stacked as Lego blocks" [16]), this technology can hardly be used for the fabrication of commercial devices. At the same time, the use of epitaxial growth techniques, in particular molecular beam epitaxy (MBE), potentially allows fabrication of 2D thin films and heterostructures with abrupt interfaces and a uniform wafer-scale film thickness. Moreover, when the epitaxial growth proceeds via van der Waals forces (the so-called van der Waals growth mode), the 2D materials can be grown on highly-mismatched substrates, even when symmetries of the grown film and substrate differ [17], which is important for integration with conventional 3D semiconductors.

The abilities of MBE for the 2D device fabrication were recently demonstrated by Yuan et al. [18], who fabricated highly efficient photodetector arrays with external quantum efficiency up to $62 \%$ and photoresponse time of $22 \mu$ using few-layer GaTe films grown by MBE on 3-inch Si wafer. The layer-by-layer growth mode has also been achieved in MBE of GaSe on mica and $\mathrm{Si}(001)$ substrates [19]. Wafer-scale $\mathrm{GaTe}_{x} \mathrm{Se}_{1-\mathrm{x}}$ films, grown by MBE on freshly cleaved mica or cleaned silicon substrates, were also reported by Liu et al. [20]. Thus, the opportunity of realization of high-quality films and heterostructures composed of 2D group III MCs by MBE has already been proved. However, due to a number of polytypes and additional phases, the synthesis of defect-free films is still a problem. It is also complicated by the much lower substrate temperatures used in MBE as compared to other epitaxial techniques.

In this paper, we present the results on MBE growth of thin films of III-VI layered MCs (GaSe, InSe, and GaTe) and their heterostructures on conventional GaAs(001) substrates, as well as studies of their structural and optical properties. The use of a combination of conventional and two-dimensional semiconductors in a single heterostructure allows expanding the range of potential applications. Although the first publications on MBE growth of group III MCs date back to the early 1990s [21,22], there are many gaps in understanding growth mechanisms, and there is still no universal recipe for the growth of high-quality optically active structures based on these layered materials.

\section{Materials and Methods}

\subsection{Materials Properties}

The layered chalcogenides of the $M X$ type, where $X=(\mathrm{Se}, \mathrm{Te})$ and $M=(\mathrm{Ga}, \mathrm{In})$, consist of vertically ordered layers which are bonded together by weak van der Waals forces, and each layer contains four covalently-bonded atomic sheets in the sequence X-M-M-X.

GaSe is one of the most studied 2D layered III-VI semiconductors, which has an indirect bandgap in the visible range with an energy of $\sim 2 \mathrm{eV}$ at $\mathrm{T}=300 \mathrm{~K}$, which is only $25 \mathrm{meV}$ lower than the direct bandgap [1,23-25]. GaSe films can crystallize in different polytypes $(\beta, \varepsilon, \gamma$, and $\delta)$, which differ from each other by stacking sequence of the layers [26]. $\gamma$-GaSe has a rhombohedral crystal lattice with the R3m space group and the lattice parameters $a=3.75 \AA$ and $c=23.92 \AA$, whereas $\beta-, \varepsilon$, and $\delta$-GaSe have a hexagonal crystal lattice with $\mathrm{P} 6_{3} / \mathrm{mmc}, \mathrm{P} 6 \mathrm{~m} 2$, and $\mathrm{P}_{3} \mathrm{mc}$ space groups, respectively [26]. Both hexagonal $\beta$ - and $\varepsilon$-GaSe polytypes have the same lattice parameters $a=3.750 \AA$ and $c=15.94 \AA$ [1] . The formation of one or another polytype depends on both the technique and growth conditions used for the GaSe film fabrication (see, e.g., [27]). In particular, GaSe layers grown by MBE are usually of $\varepsilon$ or $\gamma$-polytype $[19,28-30]$. However, some domains of $\beta$-polytype were also observed in GaSe films grown by MBE on $\mathrm{MoS}_{2}$ cleaved surfaces [31]. The thickness of one monolayer (or, more accurately, one tetralayer (TL)) GaSe is $\sim 0.8 \mathrm{~nm}$ for all polytypes.

As with many other 2D materials, GaSe is unstable and oxidizes in ambient conditions, which leads to a gradual degradation of its optical properties [32-34]. The Raman spectroscopy analysis showed that $\mathrm{GaSe}$ decomposes into $\mathrm{Ga}_{2} \mathrm{Se}_{3}$ and amorphous Se during oxidation [32,34]. Moreover, during the 
prolonged storage in air, the GaSe surface also undergoes visible transformations [35]. The oxidation of GaSe is a rapid process with a monolayer reaching an oxidized state almost immediately after exposure to air [34]. To prevent the atmospheric oxidation, the surface passivation using 50-nm-thick $\mathrm{Al}_{2} \mathrm{O}_{3}$ layer was proposed [33]. The effective protection is even more important if the grown GaSe layer consists of a large number of nanometer-scale nanoplatelets.

InSe is a direct-gap semiconductor with $\mathrm{E}_{\mathrm{g}}=1.25-1.3 \mathrm{eV}$ at room temperature in the bulk form; however, there is some spread in the bandgap values reported in the literature for various polytypes [1,36-39]. There are three polytypes of layered InSe $(\beta, \gamma$, and $\varepsilon)$, which differ in the symmetry and stacking sequence of the layers $[1,36]$. The crystal space groups of the respective InSe polytypes are the same as in the case of GaSe. The lattice constant as well as the distance between two neighboring layers are nearly the same for all polytypes: $\mathrm{a} \approx 4.0 \AA$ [36,40-42], $\mathrm{d} \approx 0.84 \mathrm{~nm}[1,36,39,43]$. The $\varepsilon$ - and $\gamma$-InSe are the most widely studied InSe polytypes with a hexagonal unit cell. These two hexagonal phases are hardly distinguishable, as they exhibit a similar composition of the unit cell and the same lattice parameters. The $\gamma$-polytype has a rhombohedral unit cell with the lattice parameter $c=24.96 \AA$, which is 1.5 times larger than that of $\beta$-InSe and $\gamma$-InSe.

In contrast to the most commonly studied $2 \mathrm{D}$ materials, the direct-gap semiconductor GaTe $\left(\mathrm{E}_{\mathrm{g}}=1.7 \mathrm{eV}\right.$ in a bulk form at $\mathrm{T}=300 \mathrm{~K}$ [1]) has a monoclinic crystal structure in the space group $\mathrm{C} 2 / \mathrm{m}$, the symmetry of which is less than hexagonal lattice structure, with lattice parameters $a=17.32 \AA$, $\mathrm{b}=4.05 \AA, \mathrm{c}=10.54 \AA$ and $\beta=104.4^{\circ}$ [1]. Low-symmetry 2D materials show significant in-plane anisotropy in their electrical, optical, and thermal properties [44]. Two-thirds of the Ga-Ga bonds in GaTe are perpendicular to the layer and the rest are parallel to the layer. The thickness of each GaTe layer is also $\sim 0.8 \mathrm{~nm}[1,18,45]$. A metastable hexagonal phase for GaTe has also been reported with the lattice parameters $\mathrm{a}=4.06 \AA$ and $\mathrm{c}=16.96 \AA$ (space group $\mathrm{P}_{3} / \mathrm{mmc}$ ) [46].

Similar to GaSe, GaTe is also characterized by a very high oxidation rate in air, which results in a gradual degradation of its optical properties. Moreover, recently Fonseca et al. demonstrated that GaTe exposure to air leads to a significant restructuring of the conduction band due to the incorporation and chemisorption of oxygen to tellurium with subsequent transformation into a disordered $\mathrm{GaTe}-\mathrm{O}_{2}$ phase [47]. To prevent the atmospheric oxidation of the $\mathrm{GaTe}$, the surface passivation with $\mathrm{Al}_{2} \mathrm{O}_{3}$ is required [33]. In this study, we stored the GaTe samples in vacuum. This method also substantially delays the degradation of optical properties, but, of course, is not practical for real-life applications.

\subsection{Thin-Films Synthesis}

Thin films of 2D-layered MCs were grown on epi-ready GaAs(001) substrates at a substrate temperature of $\mathrm{T}_{\mathrm{S}}=400-530^{\circ} \mathrm{C}$ using a double-chamber MBE setup (SemiTEq, St. Petersburg, Russia). Standard Ga, In, and Te effusion cells as well as a Se valve cracking cell (Veeco, Plainview, NY, USA) with the cracking zone temperature $\mathrm{T}_{\mathrm{Se}}(\mathrm{cr})=500{ }^{\circ} \mathrm{C}$ were used as molecular beam sources. The samples were grown either directly on thermally cleaned GaAs substrate or on 200-nm-thick GaAs buffer layer grown in a separate III-V growth chamber and transferred to the III-VI chamber through vacuum. In the former case, the substrates were first heated to $\mathrm{T}_{\mathrm{S}} \sim 600{ }^{\circ} \mathrm{C}$ without an As overpressure to remove the surface oxide layer, which was controlled by reflection high energy electron diffraction (RHEED) measurements, and then the substrate was cooled to the deposition temperature. In the latter case, after the transferring of GaAs buffer to the III-VI growth chamber the substrate temperature was gradually increased to the growth temperature with the rate $\sim 20^{\circ} \mathrm{C} / \mathrm{min}$. When the substrate temperature was stabilized at a given value, the growth was started as soon as possible to avoid the reaction between the substrate surface and residual Se. In the case when the initial deposition temperature was below $500{ }^{\circ} \mathrm{C}$, we used also the main shutter for additional protection of $\mathrm{GaAs}(001)$ growth surface. The VI/III flux ratio was controlled by measuring the beam equivalent pressures (BEPs) of the respective elements at the substrate position by using a Bayard-Alpert ion gauge. The growth parameters of GaSe and GaTe epitaxial layers are outlined below. The details of MBE growth of specific III-VI MCs and heterostructures are given in the corresponding parts of the paper. 


\subsection{Characterization}

The samples were characterized by cross-sectional transmission electron microscopy (TEM), scanning electron microscopy (SEM), X-ray diffraction (XRD), Raman spectroscopy, and photoluminescence (PL) spectroscopy techniques. The growth of all layers and heterostructures was monitored in situ by using RHEED.

The Raman measurements were performed both at room and liquid nitrogen temperatures using a T64000 (Horiba Jobin-Yvon, Lille, France) spectrometer equipped with a confocal microscope. The line at $\lambda=532 \mathrm{~nm}$ of Nd:YAG laser (Torus, Laser Quantum, Inc., Edinburg, UK) and the 514.5 and $488 \mathrm{~nm}$ lines of Argon laser (Spectra-Physics, Inc., Mountain View, CA, USA) were used as the excitation sources. The laser power on the samples was as low as $\sim 25-80 \mu \mathrm{W}$ with a spot size of $\sim 1 \mu \mathrm{m}$ in diameter. The room-temperature PL spectrum of the GaSe/GaAs(001) layer was also measured using the described experimental setup.

The low-temperature PL measurements of the $\mathrm{GaTe} / \mathrm{GaAs}(001)$ layer as well as temperature-dependent PL measurements of the InSe/GaAs(001) layer were carried out using experimental setup based on a Vertex 80 Fourier-transform infrared (FTIR) spectrometer (Bruker, Germany) equipped with a Si photodiode detector and a $\mathrm{CaF}_{2}$ beam splitter as described in Ref. [48]. The samples were placed into a Janis CCS-150 closed-cycle helium cryostat with a LakeShore 325 temperature controller, and the luminescence was extracted via $\mathrm{CaF}_{2}$ window and lenses. A $405 \mathrm{~nm}$ violet diode laser SSP-DHS-405 was used as the excitation source.

Micro-PL spectra of the GaSe/GaAs(001) layers were measured within the 10-110 K temperature range in a He-flow ST-500-Attocube cryostat. The spectra were recorded using a cooled CCD camera; the laser line at $\lambda=405 \mathrm{~nm}$ of CUBE laser (Coherent) was used as the excitation source. The laser power density on the sample was below $1 \mathrm{~W} / \mathrm{cm}^{2}$.

Time-resolved measurements of the luminescence in $\mathrm{ZnSe} / \mathrm{InSe} / \mathrm{ZnSe}$ structures were performed at $\mathrm{T}=77 \mathrm{~K}$. The samples were excited by $405 \mathrm{~nm}$ radiation of CUBE laser (Coherent) with pulses of $45 \mathrm{ps}$ duration at a repetition rate of $1 \mathrm{MHz}$.

The cross-section transmission electron microscopy (TEM) measurements were carried out using Jeol JEM-2100F microscope (the accelerating voltage $200 \mathrm{kV}$; the pixel resolution $0.19 \mathrm{~nm}$ ). The samples were prepared by the standard procedure, i.e., by mechanical polishing with subsequent thinning by etching with $\mathrm{Ar}^{+}$ions $(4 \mathrm{keV})$. D2 Phaser powder diffractometer (Bruker, Germany) was used for the XRD measurements. The SEM measurements were used to estimate the thickness of the layers and to study the surface morphology of the grown layers. Both plan-view and cross-section SEM images of the grown samples were obtained by using CamScan microscope.

\section{Results and Discussion}

\subsection{MBE Growth of $\mathrm{GaSe} / \mathrm{GaAs}(001)$}

The basic peculiarities of GaSe MBE growth on GaAs substrates were established in the 1990s [49-53]. In the last decade, a new surge of interest in GaSe (and, of course, in other 2D layered materials) has arisen because of the discovery of graphene in 2004 [54]. However, as already noted, no recipe for growing the high-quality optically active structures was developed. The GaAs(111)B substrate seems to be a natural candidate for the growth of GaSe layers because of a relatively low lattice-mismatch of $\sim 6 \%$ [21]. Indeed, the MBE growth of GaSe on a hexagonally symmetric, three-dimensional GaAs(111)B substrate has been shown to be free of the critical thickness problem, which is of a great importance for potential device applications [21,49]. However, the growth of GaSe on GaAs(111) is hardly achieved at growth temperatures above $500{ }^{\circ} \mathrm{C}$, since GaSe is re-evaporated from the growing surface due to a weak van der Waals force [49]. As a result, no PL emission from the GaSe films was detected due to its rather poor crystallinity.

The van der Waals growth mode was also realized in MBE growth of GaSe/GaAs(001) at low temperatures $\mathrm{T}_{\mathrm{S}} \approx 400{ }^{\circ} \mathrm{C}$, with the $c$ axis of the growing GaSe layer oriented along the normal to 
the substrate surface [49]. At higher growth temperatures $\left(\mathrm{T}_{\mathrm{S}}>500{ }^{\circ} \mathrm{C}\right)$, the nucleation of GaSe on the $\mathrm{GaAs}(001)$ surface occurs through the formation of transitional submonolayers with a chemical bonding between the substrate and the layer [49,50].

Both spiral and island growth modes were reported for GaSe layers grown by MBE on different substrates $[31,52,55,56]$. In particular, the spiral pyramid-like morphology has been reported for the $\mathrm{GaSe} / \mathrm{GaAs}(001)$ layers grown by $\mathrm{MBE}$ at low $\mathrm{T}_{\mathrm{S}} \approx 400{ }^{\circ} \mathrm{C}$ [55]. The evolution of GaSe morphology with growth time, observed by using the atomic force microscopy (AFM) technique, has been explained by the realizing of screw-dislocation-driven (SDD) growth mode, which is typical for the growth of 2D materials [57]. We did not use AFM in this study systematically; however, our data on GaSe/GaAs(001) layers are in a good agreement with those of other research groups. Thus, the existence of SDD growth mechanism in our GaSe layers grown at $\mathrm{T}_{\mathrm{S}} \approx 400{ }^{\circ} \mathrm{C}$ directly on GaAs $(001)$ substrates is highly likely. To grow uniform large-area layered materials with a controlled number of layers the SDD growth mode should definitely be avoided. According to Nie et al. [57], to do this, both the growth rate and the chalcogen-to-metal ratio need to be limited during deposition, and these parameters are especially important at the early nucleation and growth stages. The quality of the substrate also plays a significant role, so the use of a GaAs buffer layer along with a low growth rate as well as a choice of appropriate growth conditions could, in principle, provide the layer-by-layer growth mode in MBE of layered chalcogenides. The need for a low deposition rate for the formation of flat GaSe layers was confirmed by Yuan et al. [19], who demonstrated layer-by-layer growth of GaSe on mica and $\mathrm{Si}(001)$ substrates with a growth rate in the range $0.3-0.8 \mathrm{~nm} / \mathrm{min}$. The importance of a very low GaSe deposition rate $\left(\mathrm{r}_{\mathrm{GaSe}} \approx 0.2 \mathrm{~nm} / \mathrm{min}\right)$ for the controllable growth of high-quality atomically thin GaSe layers was also demonstrated by Chen et al. [58].

The need for a low Se/Ga flux ratio is confirmed by the experimental fact that the optimum conditions for the MBE growth of GaSe on $\mathrm{GaAs}(001)$ substrates correspond to the nearly stoichiometric ratio between the adsorbed $\mathrm{Ga}$ and Se adatoms on the growth surface $[30,49,50]$. An analysis of the available literature data, taking into account the dependence of Se incorporation coefficient on both substrate and cracking zone temperatures of the Se valve source [59], shows that the stoichiometric conditions can be estimated as corresponding to $\mathrm{P}_{\mathrm{Se}} / \mathrm{P}_{\mathrm{Ga}}(\mathrm{BEP}) \sim 12$ and $\sim 25$ at $\mathrm{T}_{\mathrm{S}}=400{ }^{\circ} \mathrm{C}$ and $500{ }^{\circ} \mathrm{C}$, respectively, if standard $\mathrm{Ga}$ and Se valve cracking cells with $\mathrm{T}_{\mathrm{Se}}(\mathrm{cr})=500{ }^{\circ} \mathrm{C}$ are used as molecular beam sources [28]. In our analysis, we used the measured $\mathrm{P}_{\mathrm{Se}} / \mathrm{P}_{\mathrm{Ga}}(\mathrm{BEP})$ flux ratios needed to obtain a relatively smooth GaSe surface at various temperatures $\left(350-450{ }^{\circ} \mathrm{C}\right)$ from [30], where a Se valve cracking cell with $\mathrm{T}_{\mathrm{Se}}(\mathrm{cr})=950^{\circ} \mathrm{C}$ was applied as a Se source, as well as data on GaSe layers grown by MBE using standard $\mathrm{Ga}$ and Se cells $[29,49-51]$. The estimated dependence of the $\mathrm{R}=\mathrm{P}_{\mathrm{Se}} / \mathrm{P}_{\mathrm{Ga}}$ (BEP) ratio, corresponding to the stoichiometric conditions on the growth surface for various $T_{S}$, is presented in Figure 1. One should note that it is difficult to associate the Se/Ga BEPs ratios taken from different sources with the stoichiometric growth conditions, especially keeping in mind that there is some uncertainty in determining the real temperature of epitaxial growth. However, it can be assumed that the formation of gallium droplets on the GaSe surface is the only reliable indicator of Ga-rich MBE growth conditions. The points corresponding to the GaSe layers grown at different $\mathrm{T}_{\mathrm{S}}$ with and without Ga droplets on the growth surface are shown in Figure S1. The obtained data confirm the correctness of our estimations. It follows directly from the dependence in Figure 1 that the stoichiometry on the growth surface at a fixed $\mathrm{T}_{\mathrm{S}}$ can be controlled either by adjusting the opening of the Se valve or by changing the cracking zone temperature $\mathrm{T}_{\mathrm{Se}}(\mathrm{cr})$ of the valve Se cell. In the latter case, the change in the ratio of the Se and Ga adatoms on the growth surface occurs due to a change in the Se sticking coefficient.

The growth of GaSe on GaAs(001) substrates was initiated by the simultaneous opening of Ga and Se fluxes. One should note, the RHEED observations showed that initial $(2 \times 4)$ As reconstruction of the $\mathrm{GaAs}(001)$ buffer layer changed to the $(2 \times 1)$ Se-terminated one while rising the substrate temperature under Se exposure or unintentionally if there was a Se background in the growth chamber. The growth 
parameters as well as the thicknesses of the GaSe epitaxial layers are outlined in Table 1. The presence or absence of a buffer layer in a particular structure is also indicated.

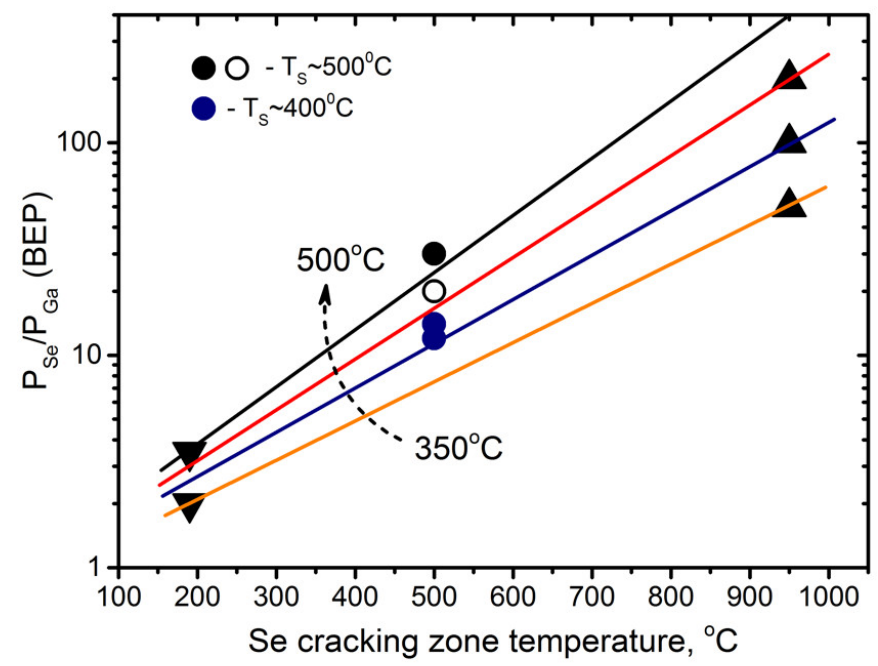

Figure 1. The calculated dependences of the $\mathrm{P}_{\mathrm{Se}} / \mathrm{P}_{\mathrm{Ga}}(\mathrm{BEP})$ ratio corresponding to stoichiometric conditions on the GaSe growth surface at different $\mathrm{T}_{\mathrm{S}}$, marked in different colors $\left(\mathrm{T}_{\mathrm{S}}=350{ }^{\circ} \mathrm{C}\right.$, orange; $\mathrm{T}_{\mathrm{S}}=400{ }^{\circ} \mathrm{C}$, blue; $\mathrm{T}_{\mathrm{S}}=450{ }^{\circ} \mathrm{C}$, red; and $\mathrm{T}_{\mathrm{S}}=500{ }^{\circ} \mathrm{C}$, black). The data of Lee et al. [30] are shown by filled triangles with the vertex pointing up $\left(\mathrm{T}_{\mathrm{Se}}(\mathrm{cr})=950{ }^{\circ} \mathrm{C}\right)$. The data on $\mathrm{P}_{\mathrm{Se}} / \mathrm{P}_{\mathrm{Ga}}(\mathrm{BEP})$ flux ratios corresponding to nearly stoichiometric conditions for GaSe layers growth using standard Ga and Se cells are shown by filled triangles with the vertex pointing down. Our data on $\mathrm{P}_{\mathrm{Se}} / \mathrm{P}_{\mathrm{Ga}}(\mathrm{BEP})$ flux ratio for the GaSe layers grown at $\mathrm{T}_{\mathrm{S}}=400$ and $500{ }^{\circ} \mathrm{C}$ are shown by blue and black circles, respectively. GaSe layers with and without Ga droplets on the growth surface are indicated by the hollow and filled circles.

Table 1. The growth parameters of GaSe/GaAs(001) layers.

\begin{tabular}{|c|c|c|c|c|c|c|}
\hline Layer & $\mathrm{T}_{\mathrm{Ga}} ; \mathrm{Ga}$ flux (BEP) & $\begin{array}{c}\text { Se/Ga Flux } \\
\text { Ratio }{ }^{1}, \\
\text { (BEP/Real) }\end{array}$ & $T_{\mathrm{S}},{ }^{\circ} \mathrm{C}$ & $\begin{array}{c}\text { Layer } \\
\text { Thickness, nm } \\
\text { (SEM) }\end{array}$ & $\begin{array}{c}\text { Average } \\
\text { Growth Rate }{ }^{2}, \\
\text { nm/min }\end{array}$ & Notes \\
\hline \#GS1 & $\begin{array}{c}\mathrm{T}_{\mathrm{Ga}}=910^{\circ} \mathrm{C} ; \\
\mathrm{P}_{\mathrm{Ga}}=4.7 \times 10^{-8} \text { Torr }\end{array}$ & $\begin{array}{l}\mathrm{Se} / \mathrm{Ga}(\mathrm{BEP}) \sim 34 ; \\
\quad(\mathrm{Se} / \mathrm{Ga} \sim 3.9)\end{array}$ & 410 & 200 & 1.3 & $\begin{array}{l}\text { Se cracking zone } \\
\text { temperature } \\
T_{S e}(\mathrm{cr})=450{ }^{\circ} \mathrm{C}\end{array}$ \\
\hline \#GS2 & $\begin{array}{c}\mathrm{T}_{\mathrm{Ga}}=900^{\circ} \mathrm{C} ; \\
\mathrm{P}_{\mathrm{Ga}}=4.0 \times 10^{-8} \text { Torr }\end{array}$ & $\begin{array}{l}\mathrm{Se} / \mathrm{Ga}(\mathrm{BEP}) \sim 42 ; \\
\quad(\mathrm{Se} / \mathrm{Ga} \sim 4.8)\end{array}$ & 400 & 270 & 1.13 & $\begin{array}{l}\text { Se cracking zone } \\
\text { temperature } \\
T_{S e}(\mathrm{cr})=450{ }^{\circ} \mathrm{C}\end{array}$ \\
\hline \#GS3 & $\begin{array}{c}\mathrm{T}_{\mathrm{Ga}}=940^{\circ} \mathrm{C}(1 \text { st stage }) ; \\
\mathrm{T}_{\mathrm{Ga}}=920^{\circ} \mathrm{C}(\text { 2nd stage }) ; \\
\mathrm{P}_{\mathrm{Ga}}=9.0 \times 10^{-8} \text { Torr }(1 \text { st stage }) \\
\mathrm{P}_{\mathrm{Ga}}=6.0 \times 10^{-8} \text { Torr (2nd stage) }\end{array}$ & $\begin{array}{c}\mathrm{Se} / \mathrm{Ga} \\
(\mathrm{BEP}) \sim 20 / 30 \\
(\mathrm{Se} / \mathrm{Ga} \sim 1.6 / 1.2)\end{array}$ & $\begin{array}{l}\text { 410-1st stage } \\
500-2 \text { nd stage }\end{array}$ & 360 & $\sim 3$ & $\begin{array}{l}0.2-\mu \mathrm{m} \text { thick GaAs } \\
\text { buffer layer; } \\
\text { two-stage growth } \\
\text { mode }\end{array}$ \\
\hline \#GS4 & $\begin{array}{c}\mathrm{T}_{\mathrm{Ga}}=940^{\circ} \mathrm{C} ; \\
\mathrm{P}_{\mathrm{Ga}}=9.0 \times 10^{-8} \text { Torr }\end{array}$ & $\begin{array}{c}\mathrm{Se} / \mathrm{Ga} \\
(\mathrm{BEP}) \sim 18-20 \\
(\mathrm{Se} / \mathrm{Ga} \sim 0.9-0.95)\end{array}$ & 480 & 50 & $\sim 1.4$ & $\begin{array}{l}\text { Ga droplets on the } \\
\text { surface with diameter } \\
\sim 200-300 \mathrm{~nm}\end{array}$ \\
\hline \#GS5 & $\begin{array}{c}\mathrm{T}_{\mathrm{Ga}}=954^{\circ} \mathrm{C} ; \\
\mathrm{P}_{\mathrm{Ga}}=1.25 \times 10^{-7} \text { Torr }\end{array}$ & $\begin{array}{c}\mathrm{Se} / \mathrm{Ga} \\
(\mathrm{BEP}) \sim 12-13 \\
(\mathrm{Se} / \mathrm{Ga} \sim 1)\end{array}$ & 410 & 300 & 5 & $\begin{array}{l}0.2-\mu \mathrm{m} \text { thick GaAs } \\
\text { buffer layer }\end{array}$ \\
\hline \#GS6 & $\begin{array}{c}\mathrm{T}_{\mathrm{Ga}}=910^{\circ} \mathrm{C} ; \\
\mathrm{P}_{\mathrm{Ga}}=5.5 \times 10^{-8} \text { Torr }\end{array}$ & $\begin{array}{c}\mathrm{Se} / \mathrm{Ga} \\
(\mathrm{BEP}) \sim 13-14 \\
(\mathrm{Se} / \mathrm{Ga} \sim 1.1-1.2)\end{array}$ & 405 & 90 & 1.5 & \\
\hline \#GS7 & $\begin{array}{c}\mathrm{T}_{\mathrm{Ga}}=910^{\circ} \mathrm{C} ; \\
\mathrm{P}_{\mathrm{Ga}}=5.5 \times 10^{-8} \text { Torr }\end{array}$ & $\begin{array}{l}\mathrm{Se} / \mathrm{Ga}(\mathrm{BEP}) \sim 25 ; \\
\quad(\mathrm{Se} / \mathrm{Ga} \sim 1)\end{array}$ & 500 & 400 & 2.2 & \\
\hline \#GS8 & $\begin{array}{c}\mathrm{T}_{\mathrm{Ga}}=890^{\circ} \mathrm{C} ; \\
\mathrm{P}_{\mathrm{Ga}}=3.8 \times 10^{-8} \text { Torr }\end{array}$ & $\begin{array}{l}\mathrm{Se} / \mathrm{Ga}(\mathrm{BEP}) \sim 21 ; \\
\quad(\mathrm{Se} / \mathrm{Ga} \sim 1.6)\end{array}$ & 410 & - & $\sim 1.0$ & rough growth surface \\
\hline
\end{tabular}

${ }^{1}$ The calculated Se/Ga ratios of the impinging atoms on the GaAs surface are given taking into account the growth temperature $\left(T_{S}\right)$ and the cracking zone temperature of the valve Se source, influencing the Se sticking coefficient. 2 The average growth rate was calculated using the layer thickness determined from SEM measurements and deposition time. 
The surface morphology of the GaSe/GaAs(001) layers was analyzed by SEM. The plan-view images of the thick $\left(>50 \mathrm{~nm}\right.$ ) layers grown at $\mathrm{T}_{\mathrm{S}} \sim 400{ }^{\circ} \mathrm{C}$ and at different Se/Ga flux ratios with nearly the same growth rate of $\sim 1.1-1.4 \mathrm{~nm} / \mathrm{min}$ look quite similar: one can see a number of so-called "nanoplatelets" of different size and shape, randomly distributed on the relatively flat growth surface (Figure 2a,b). The similar surface morphology was observed in GaSe layers grown on GaAs(112) substrates at the same growth temperature $\mathrm{T}_{\mathrm{S}} \sim 400{ }^{\circ} \mathrm{C}$ [28]. At the optimized conditions, where the adsorbed $\mathrm{Ga}$ and Se adatoms are close to stoichiometry, and the growth rate is relatively low, the RHEED pattern remained streaky for a long-time during MBE growth (Figure S2a). However, under strong Se-rich conditions the brightness of the RHEED pattern gradually decreased with increasing layer thickness, and the streaky reflections became wider and blurred. In some areas of the RHEED pattern, spots as well as Debye rings appeared, indicating a polycrystalline growth. The observed RHEED images are very similar to that reported in [30] for the GaSe layers grown on GaN/sapphire in Se-rich conditions, and reflect an increase in surface roughness as the layer grows, which can be induced by the formation of a multi-domain structure at the initial growth stage. We assume that the "growth window" in the case of MBE growth of GaSe using standard Ga and Se valve cracking cells as molecular beam sources is narrower than in the case of using standard Ga and Se effusion cells.

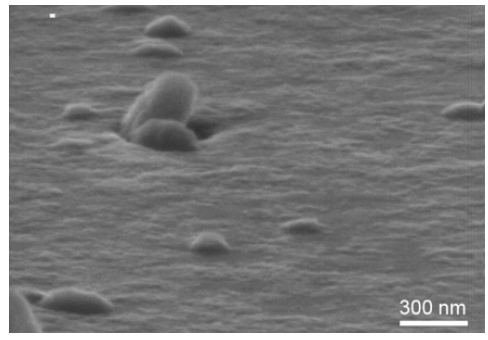

(a)

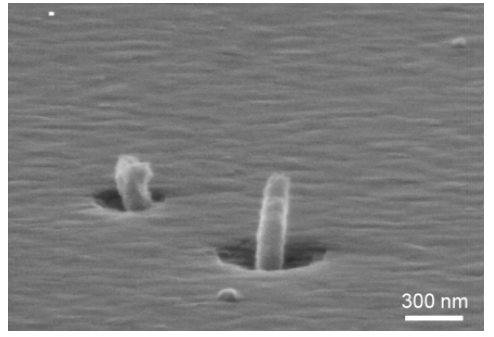

(b)

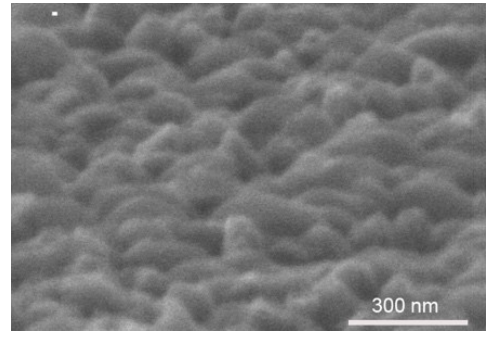

(c)

Figure 2. SEM images of the GaSe layers grown on the epi-ready GaAs(001) substrates at $\mathrm{T}_{\mathrm{S}} \sim 400{ }^{\circ} \mathrm{C}$. The Se/Ga (BEP) flux ratios and growth rates were the following: (a) Se/Ga (BEP) 42, $\mathrm{r}_{\mathrm{GaSe}} \approx 1.13 \mathrm{~nm} / \mathrm{min}$, sample \#GS2; (b) Se/Ga(BEP) 34, $\mathrm{r}_{\mathrm{GaSe}} \approx 1.3 \mathrm{~nm} / \mathrm{min}, \# G S 1$; (c) Se/Ga $(\mathrm{BEP}) \sim 12-13, \mathrm{r}_{\mathrm{GaSe}} \approx 5 \mathrm{~nm} / \mathrm{min}$, \#GS5. The thicknesses of the structures ranged within 200-300 $\mathrm{nm}$. In the case of using standard Ga and Se valve cracking cell with $\mathrm{T}_{\mathrm{Se}}(\mathrm{cr})=500{ }^{\circ} \mathrm{C}$ as molecular beam sources, the stoichiometric Se/Ga ratio at $\mathrm{T}_{\mathrm{S}}=400{ }^{\circ} \mathrm{C}$ corresponds to the $\mathrm{P}_{\mathrm{Se}} / \mathrm{P}_{\mathrm{Ga}}(\mathrm{BEP}) \sim 12$.

Although the Se/Ga flux ratio affects both the surface density of nanoplatelets and their size, the main parameter determining the surface morphology, in addition to $\mathrm{T}_{\mathrm{S}}$, is the Ga flux which controls the GaSe growth rate. The SEM image of the GaSe layer grown with a deposition rate of $\sim 5 \mathrm{~nm} / \mathrm{min}$ at $\mathrm{T}_{\mathrm{S}} \sim 400{ }^{\circ} \mathrm{C}$, as shown in Figure $2 \mathrm{c}$, reveals a very rough surface of GaSe film despite the nearly stoichiometric flux ratio used. We suppose that the mobility of gallium atoms in this case is apparently insufficient for the formation of the flat layer morphology.

The cross-section TEM image of the GaSe layer grown at $\mathrm{T}_{\mathrm{S}} \sim 400{ }^{\circ} \mathrm{C}$ with a low growth rate $\mathrm{r}_{\mathrm{GaSe}} \approx 1.13 \mathrm{~nm} / \mathrm{min}$ (sample \#GS1) confirms the formation of a relatively flat layer with a nearly abrupt GaSe/GaAs(001) interface (Figure 3a). The thickness of the GaSe film is about $200 \mathrm{~nm}$ and relatively uniform. The inhomogeneous contrast of the image can be related to the high density of extended defects and a number of domains in the grown film. Figure $3 \mathrm{~b}$ shows the high-resolution TEM image of a GaSe/GaAs(001) interface for the same layer. The rough boundary between GaSe and GaAs is likely due to a thermal cleaning of the $\mathrm{GaAs}(001)$ substrate in the absence of the As flux. One can clearly see the layered structure of GaSe. The observed interplanar spacing of the film is of $\sim 0.8 \mathrm{~nm}$, which is equal to the thickness of one tetralayer. The selected area electron diffraction (SAED) (Figure S3a) revealed that the layer has a predominantly rhombohedral structure, which corresponds to the $\gamma$-polytype. The formation of the $\gamma$-GaSe polytype is typical for the MBE grown GaSe/GaAs(001) layers, as reported previously $[29,50]$. The $c$ axis of the GaSe layer is normal to the substrate/layer interface (Figure 3b). It is difficult to draw an unambiguous conclusion about the formation of any transition layer at the 
GaSe/GaAs(001) interface from the TEM image shown in Figure 3b. Nevertheless, the existence of built-in electric fields generated at the $\mathrm{GaSe} / \mathrm{GaAs}(001)$ heterointerface, as indicated by clearly observed Franz-Keldysh oscillations in the photoreflectance spectra [60], may indirectly indicate the formation of such layers at the initial growth stages. Another possible explanation is related to the diffusion of Se atoms into the GaAs substrate. The possibility of the formation of $\mathrm{Ga}_{2} \mathrm{Se}_{3}$-type intermediate layers at the GaSe/GaAs(001) interface was demonstrated by Dai et al. [29,61].

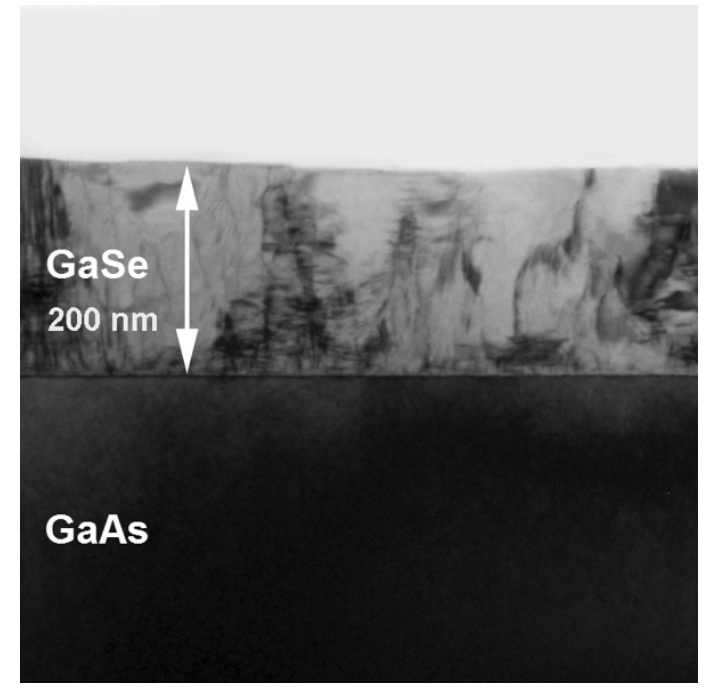

(a)

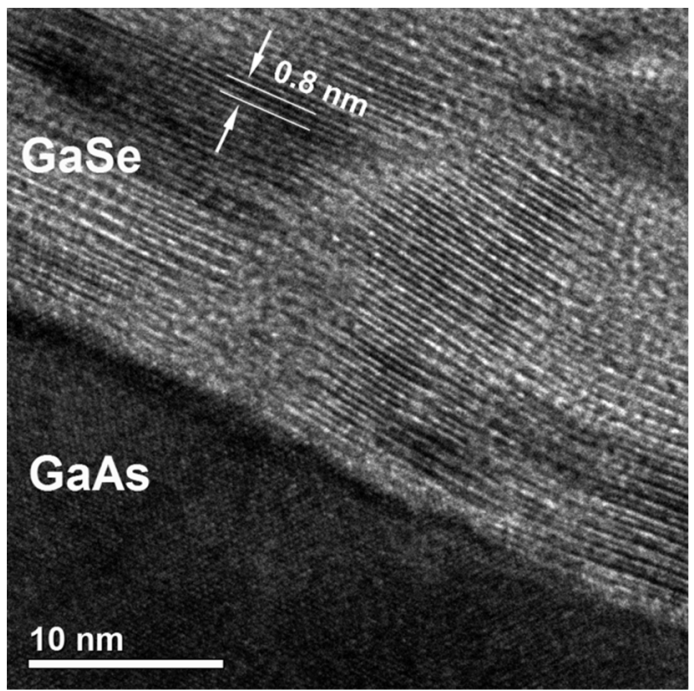

(b)

Figure 3. (a) Cross-section TEM image of the GaSe layer grown on the GaAs(001) substrate at $\mathrm{T}_{\mathrm{S}} \sim 400{ }^{\circ} \mathrm{C}$ (Se/Ga(BEP) 34) (sample \#GS1); (b) high-resolution TEM (HRTEM) image of the GaSe/GaAs(001) heterointerface for the same layer.

With increasing $\mathrm{T}_{\mathrm{S}}$, the surface morphology of the GaSe/GaAs(001) layers became rougher. It should be noted that this tendency was also observed for the GaSe layers grown in a two-stage regime, i.e., for layers in which the initial GaSe layer was deposited at a low substrate temperature $\mathrm{T}_{\mathrm{S}} \sim 400{ }^{\circ} \mathrm{C}$. At $\mathrm{T}_{\mathrm{S}} \sim 500{ }^{\circ} \mathrm{C}$, no flat layer was observed; the structure comprised an array of nanoplatelets tilted toward both [110] and [110] directions of the GaAs substrate (Figure 4a and Figure S4a) [49]. Despite a certain degree of disordering, due to the chemical interaction between the substrate and the growing layer, the $c$ axis of GaSe nanoplatelets is predominantly oriented along the $<111>$ directions of the GaAs substrate (Figure $4 b$ ). One can also see the formation of an intermediate layer near the GaSe/GaAs interface in some nanoplatelets, which leads to an additional layer tilt caused by a partial stress relaxation to accommodate the lattice mismatch between GaSe and GaAs, as previously reported by Kojima et al. [50]. Nevertheless, the areas where the GaSe domains start to grow with the $c$ axis normal to the substrate/layer interface are retained (Figure $\mathrm{S} 4 \mathrm{~b}$ ). The average growth rate of the GaSe layer shown in Figure 4 was as high as $\sim 2.2 \mathrm{~nm} / \mathrm{min}$. The appearance of the clearly resolved spots on the streaked RHEED pattern reflected the disordering on the growth surface. Nevertheless, the RHEED pattern remained nearly streaky during all $3 \mathrm{~h}$ of growth, and the brightness of the RHEED pattern remained virtually unchanged with increasing layer thickness (Figure S2b). When the growth temperature was raised up to $\mathrm{T}_{\mathrm{S}}>530-540{ }^{\circ} \mathrm{C}$, no growth of GaSe was observed even at a high Ga flux intensity.

For the GaSe layers grown at $\mathrm{T}_{\mathrm{S}} \sim 400^{\circ} \mathrm{C}$ with a Ga beam flux of $(4-6) \times 10^{-8}$ Torr, the growth rate estimated from the cross-section SEM images is nearly proportional to the impinging Ga flux, which reflects the growth of the layers with a relatively planar surface morphology (samples \#GS1, \#GS2, and \#GS6 in Table 1). However, for the layers with rough surface morphology grown at a high Ga beam flux, and for the layers consisting of a set of inclined nanoplatelets (\#GS7), the average growth 
rate estimated from cross-section SEM images is higher than the expected one from the impinging Ga flux, which confirms the growth of discontinuous layers.

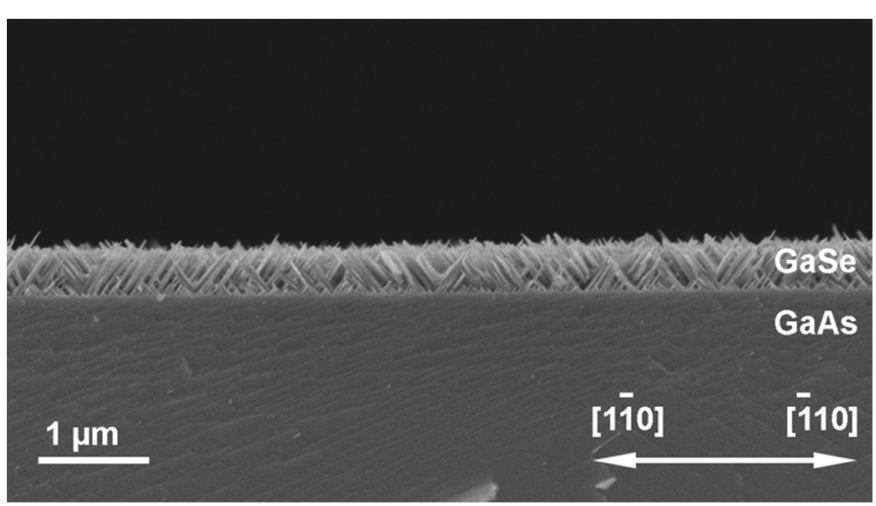

(a)

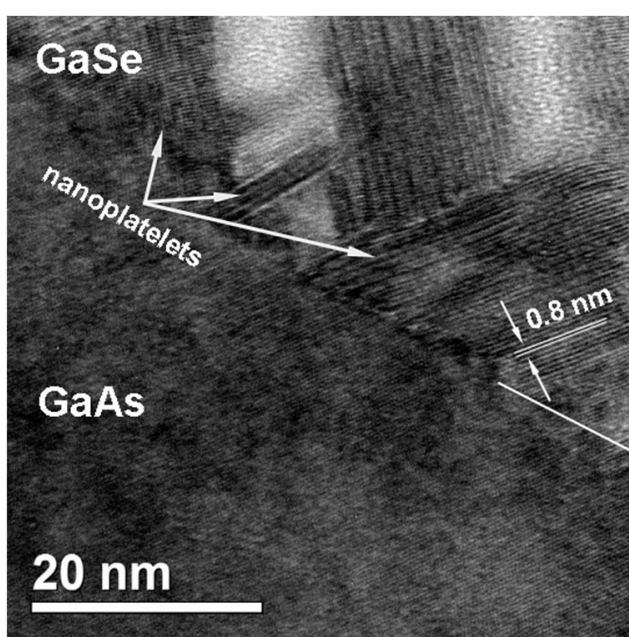

(b)

Figure 4. Cross-section SEM (a) and HRTEM (b) images of the GaSe layer grown on a GaAs(001) substrate at $\mathrm{T}_{\mathrm{S}} \sim 500{ }^{\circ} \mathrm{C}(\mathrm{Se} / \mathrm{Ga}(\mathrm{BEP}) \sim 25)$ (sample \#GS7).

The X-ray powder diffraction patterns of the studied GaSe/GaAs(001) layers are presented in Figure 5. XRD patterns in addition to the reflections from the (002) and (004) planes of GaAs substrate demonstrate the reflections from GaSe planes oriented perpendicular to the $c$ axis, which indicates the preferential orientation of the $c$ axis in the direction normal to the growth surface. A weak additional peak in the angle range $\sim 28.4^{\circ}$ along the $2 \theta$ axis on XRD patterns of the layers grown under strong Se-rich conditions (Curves 1 and 2), can be attributed to the inclusions of a $\mathrm{Ga}_{2} \mathrm{Se}_{3}$ phase [30], since its position is in good agreement with the $\mathrm{Ga}_{2} \mathrm{Se}_{3}(111)$ reflection [62], and the intensity is higher for the layer grown at the higher Se/Ga flux ratio. The preferred orientation of the $\mathrm{Ga}_{2} \mathrm{Se}_{3}$ phase is due to the proximity of interatomic distances in the $\mathrm{GaSe}(0001)$ and $\mathrm{Ga}_{2} \mathrm{Se}_{3}(111)$ planes (i.e., along GaSe $\langle 1000\rangle$ and $\mathrm{Ga}_{2} \mathrm{Se}_{3}\langle 110\rangle$ directions), being 3.755 and $3.83 \AA$, respectively. According to Afifi et al. [62], the lattice constant of cubic $\alpha-\mathrm{Ga}_{2} \mathrm{Se}_{3}$, determined from the analysis of the XRD patterns, is a $=5.44 \AA$. The existence of the $\mathrm{Ga}_{2} \mathrm{Se}_{3}$ phase inclusions in the GaSe layers grown under strong Se-rich conditions was also confirmed directly by TEM (Figure S3b).

In contrast to that, XRD patterns of the samples grown at nearly stoichiometric conditions (Curves 3-5 in Figure 5) demonstrate the absence of the $\mathrm{Ga}_{2} \mathrm{Se}_{3}$ peak. The XRD patterns of the GaSe layers, which possess rough surface morphology (Curves 3 and 5 in Figure 5), contain additional peaks that can be interpreted as reflections from the inclined GaSe planes. The appearance of these additional peaks in the X-ray diffraction pattern probably evidences the internal ordering or, in other words, indicates the existence of preferred tilt angles of the nanoplatelets.

The GaSe layers were studied also by Raman spectroscopy techniques. Figure 6 shows the Raman spectrum of the bulk $\varepsilon$-GaSe sample produced by HQ Graphene (Groningen, the Netherlands) (the bottom curve), which was measured in a scattering configuration with the incident light directed along the $c$ axis, as well as the typical Raman spectra of the GaSe/GaAs(001) epilayers grown at different conditions. The Raman spectra were measured under $532 \mathrm{~nm}$ laser excitation. It can be seen that, in the spectra of all samples, in addition to the lines corresponding to vibrational modes in GaSe [63], there are two extra lines at frequencies of 268 and $291 \mathrm{~cm}^{-1}$, which are associated with light scattering by transverse (TO) and longitudinal (LO) phonons in the GaAs substrate. However, in contrast to the spectrum of bulk $\varepsilon$-GaSe containing only one line near $213 \mathrm{~cm}^{-1}$, a distinct doublet is observed in this spectral region in the spectra of the GaSe layers grown at $\mathrm{T}_{\mathrm{S}} \sim 500{ }^{\circ} \mathrm{C}$ (Curves 4 and 5), as well as in the spectrum of the GaSe layer with the rough surface morphology grown at $\mathrm{T}_{\mathrm{S}} \sim 400{ }^{\circ} \mathrm{C}(\mathrm{Curve} 3$, 
\#GS8). According to the selection rules $[63,64]$, the line in the doublet, which we interpret as $E^{\prime \prime}$ mode, should appear in the spectrum, provided that the incident light is not directed strictly along the $c$ axis. Thus, the presence of such a doublet is an indication of the substantial deviation of the $c$ axis in the GaSe nanoplatelets from the normal to the substrate/layer interface. In addition, a broad peak with a frequency of about $250 \mathrm{~cm}^{-1}$ is observed in the spectra of the same samples. There are several explanations for its origin. This peak can be an $\mathrm{E}^{\prime}(\mathrm{LO})$ mode from $\varepsilon$-GaSe, which manifests itself in the spectra as a result of the deviation of the $c$ axis in the GaSe nanoplatelets from the normal to the substrate/layer interface, or it can arise due to symmetry breaking caused by imperfection of the crystal lattice $[65,66]$. On the other hand, this peak can also be associated with amorphous Se, which can exist on the surface, since GaSe is easily oxidized [32,67]. Thus, the obtained Raman data presented by Curves $3-5$ in Figure 6 allow us to conclude that these layers predominantly possess an $\varepsilon$-GaSe polytype. The SAED measurements of the sample \#GS3 agree well with this assumption, indicating the hexagonal crystal structure of the grown layer. An additional argument in favor of this interpretation is the first-principle calculations which predict the $\varepsilon$-polytype being a more stable form in comparison with that of $\beta$-polytype also having a hexagonal crystal structure $[65,68]$.

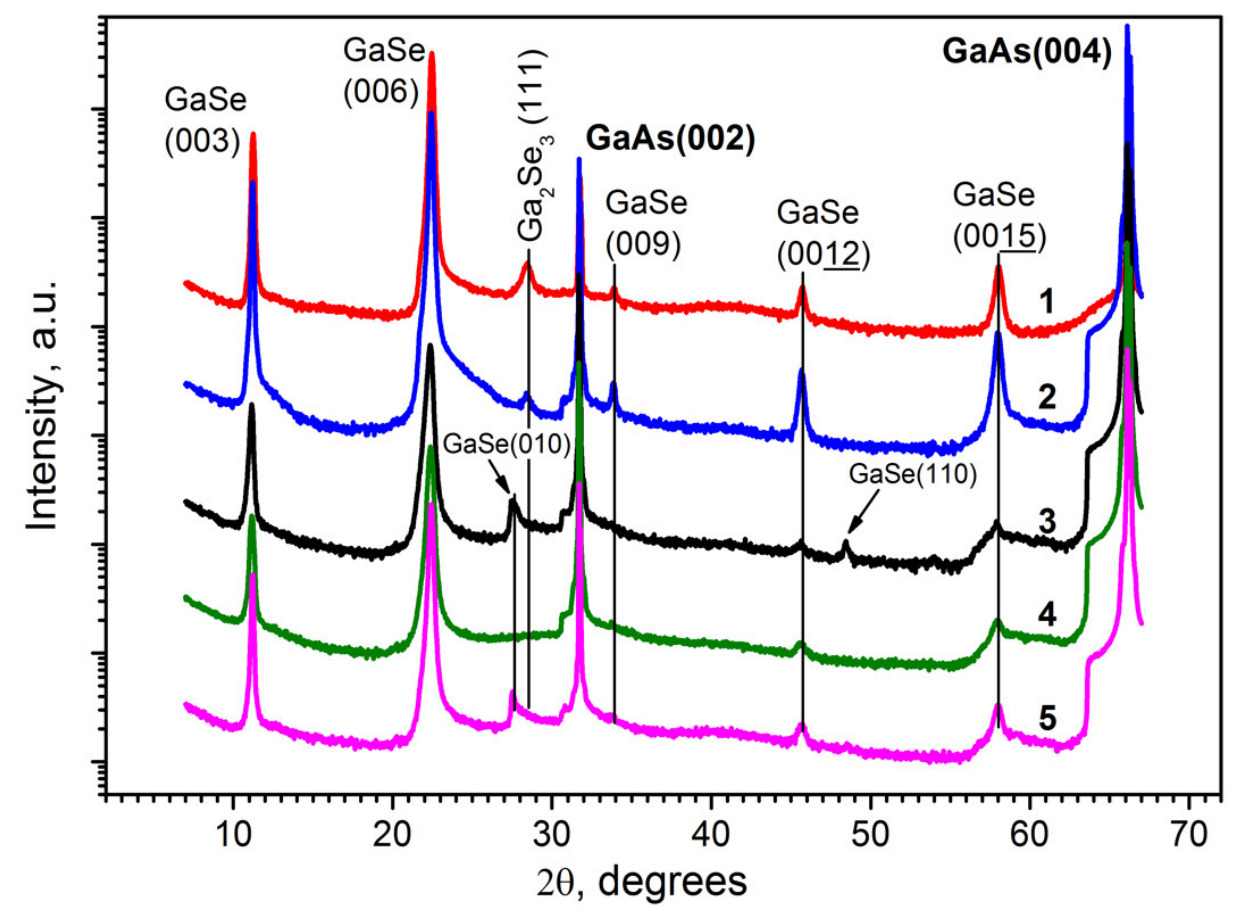

Figure 5. The X-ray powder diffraction patterns of the GaSe layers grown on GaAs(001) substrates. A weak additional peak in the angle range $\sim 28.4^{\circ}$, along the $2 \theta$ axis on XRD patterns of the layers grown under strong Se-rich conditions at $\mathrm{T}_{\mathrm{S}}=400{ }^{\circ} \mathrm{C}$ (Curves 1 and 2), is attributed to the inclusions of a $\mathrm{Ga}_{2} \mathrm{Se}_{3}$ phase. Curves 1-5 correspond to samples \#GS2, \#GS1, \#GS5, \#GS4, and \#GS3, respectively.

Curve 2 in Figure 6 shows the typical Raman spectra of flat GaSe layers grown at $\mathrm{T}_{\mathrm{S}} \sim 400{ }^{\circ} \mathrm{C}$ under strong Se-rich conditions (sample \#GS1). One can see that the fundamental difference between this spectrum and the spectrum of bulk $\varepsilon$-GaSe is the absence of a line at a frequency of $19 \mathrm{~cm}^{-1}$. According to the group-theory analysis, the lowest frequency line in the Raman spectrum of GaSe should correspond to interlayer vibrations, and therefore should not be observed in the first-order Raman spectra of $\gamma$-GaSe because the primitive unit cell of $\gamma$-GaSe contains only one layer per unit cell [69]. Another peculiarity of Curve 2 in Figure 6 is that two lines in the spectrum are shifted to low frequencies compared to similar lines in the spectrum of bulk $\varepsilon$-GaSe, which are located at frequencies of 134 and $214 \mathrm{~cm}^{-1}$ (see also Figure S5). A similar shift of the lines was reported also by Yuan et al. [19], where the correspondence of the grown layer to the $\gamma$-GaSe polytype was determined using TEM. 
An analysis of the Raman data for both $\varepsilon$ - and $\gamma$-polytypes of GaSe presented by Hoff et al. [64] also leads to the conclusion that the low-frequency shift of the two phonon modes is a characteristic of the $\gamma$-GaSe polytype. Thus, the peculiarities in the Raman spectrum of the GaSe layer grown at $\mathrm{T}_{\mathrm{S}} \sim 400^{\circ} \mathrm{C}$ (Curve 2 in Figure 6) confirm the conclusion drawn earlier from SAED (Figure S3a) that sample \#GS1 is the $\gamma$-GaSe polytype. Summarizing, from the analysis of the Raman spectra of GaSe samples, we can conclude that the formation of a particular GaSe-polytype is determined not only by the epitaxial growth temperature, but rather by a combination of several factors.

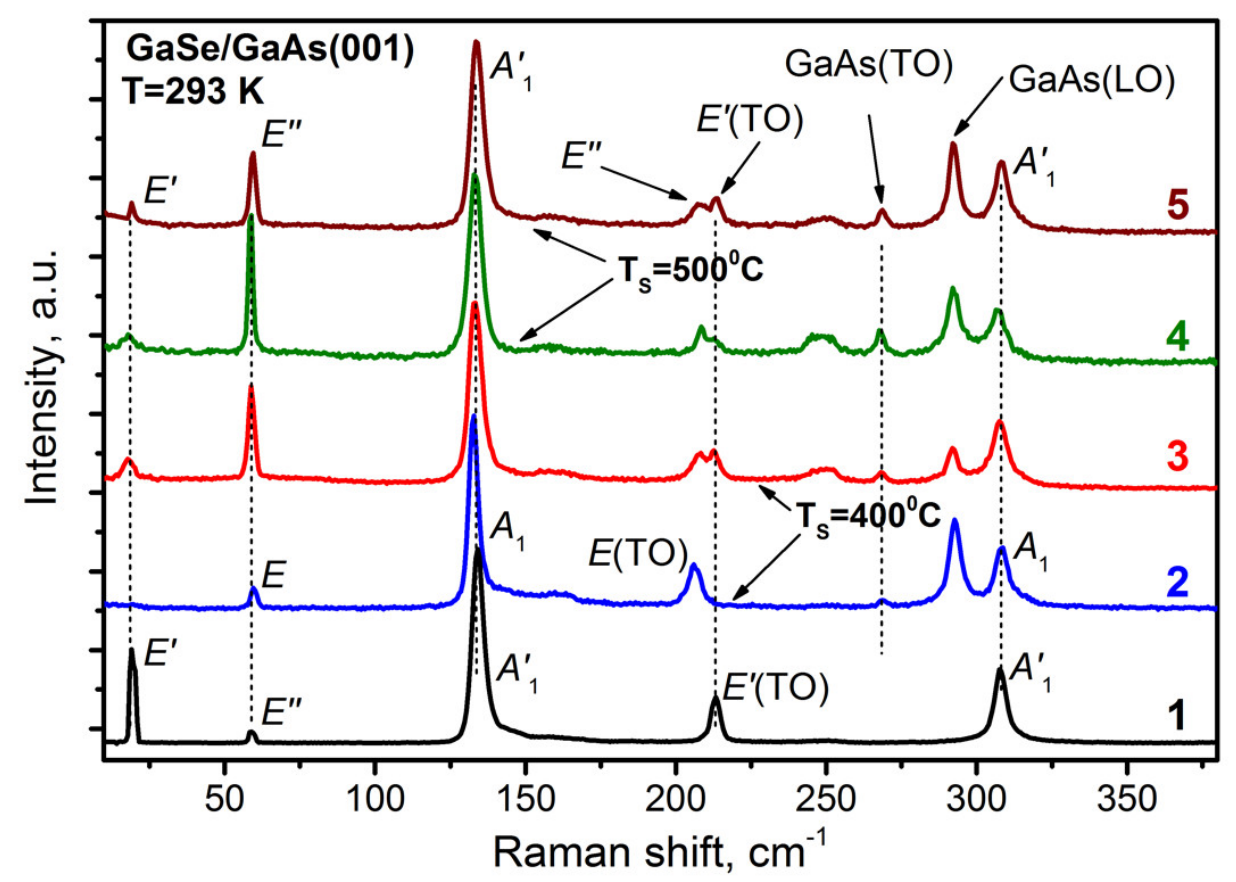

Figure 6. Raman spectrum of the bulk $\varepsilon$-GaSe layer produced by HQ Graphene (Curve 1). Raman spectra of the GaSe layers grown on $\mathrm{GaAs}(001)$ substrates at $\mathrm{T}_{\mathrm{S}} \sim 400{ }^{\circ} \mathrm{C}$ (Curves 2 and 3, samples \#GS1 and \#GS8, respectively), at $\mathrm{T}_{\mathrm{S}} \sim 500{ }^{\circ} \mathrm{C}$ (Curve 5, sample \#GS7), and using a two-stage growth mode (Curve 4, sample \#GS3). All spectra are normalized to the intensity of the $\mathrm{A}_{1}$ phonon line $\left(132 \mathrm{~cm}^{-1}\right)$.

Figure 7a demonstrates the temperature-dependent PL spectra of the $\gamma$-polytype GaSe layer grown at $T_{S}=410{ }^{\circ} \mathrm{C}$ in Se-rich regime (sample \#GS1). As can be seen in the figure, PL spectra at different temperatures demonstrate broad emission bands centered at around $1.7 \mathrm{eV}$. The peak near $1.5 \mathrm{eV}$ is attributed to the emission from GaAs substrate. The main peak at $1.7 \mathrm{eV}$ apparently consists of at least two peaks and its intensity rapidly quenches with increasing temperature. The observed PL spectra are very similar to those reported by Diep et al. for the $\mathrm{GaSe} / \mathrm{GaAs}(001)$ layers also grown at $\mathrm{T}_{\mathrm{S}}=400{ }^{\circ} \mathrm{C}$ [55], where the PL peaks were attributed to the free and bound excitons, respectively, and the strong redshift in PL was explained by the strain-induced reduction of the GaSe bandgap energy due to the SDD growth mode. The MBE growth conditions of the GaSe layers in [55] are similar to those used for growing sample \#GS1 (see Table 1), where the occurrence of SDD growth mode is definitely highly likely due to the high Se/Ga ratio. However, when tensile strain is applied a noticeable shift of both $A_{1}$ compressional modes corresponding to the stretching vibrations of the atoms and located at frequencies of $134 \mathrm{~cm}^{-1}$ and $307 \mathrm{~cm}^{-1}$ should be observed [70,71]. Nevertheless, one can see no significant shift of the $307 \mathrm{~cm}^{-1}$ line in comparison with that of the bulk sample (Curves 1 and 2 in Figure 6). In addition, the observed rapid decay of the PL intensity with temperature allowed assuming the defect-related nature of this PL band. This assumption is supported by the fact that an intense emission band centered at $1.71 \mathrm{eV}(77 \mathrm{~K})$ was observed by Capozzi and Montagna [72] in the PL spectra of "poor-quality" GaSe samples grown from the melt by using the Bridgman-Stockbarger method. For each temperature studied, this emission band was more intense and broader in the 
samples containing a higher density of structural defects or impurities with respect to the samples of good quality. Capozzi and Montagna proposed that this band originates from the recombination of free electrons, mainly at the indirect minimum, with neutral-acceptor levels [72]. A similar PL emission band was also observed in the PL spectra of $\mathrm{GaSe} / \mathrm{GaAs}(001)$ layers grown at high $\mathrm{T}_{\mathrm{S}}>500{ }^{\circ} \mathrm{C}[49,73]$. To make a reliable conclusion about the origin of the $1.7 \mathrm{eV}$ peak in the PL spectrum of our GaSe layer \#GS1, time-resolved PL measurements should definitely be carried out.

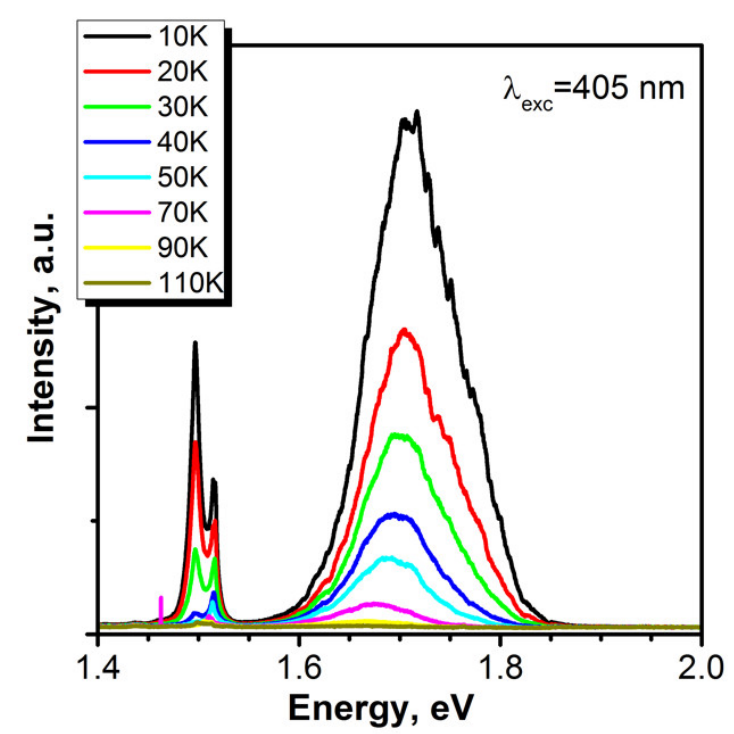

(a)

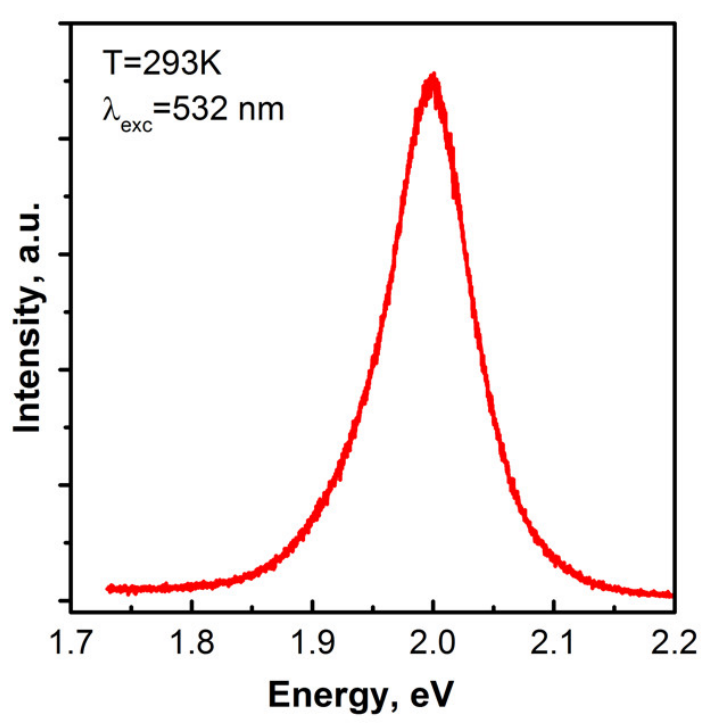

(b)

Figure 7. (a) $\mu$-PL spectra of the GaSe layer grown at $\mathrm{T}_{\mathrm{S}}=400{ }^{\circ} \mathrm{C}$ as a function of temperature (sample \#GS1). The laser power density on the sample was below $1 \mathrm{~W} / \mathrm{cm}^{2}$. (b) PL spectrum of the GaSe layer grown at $\mathrm{T}_{\mathrm{S}}=500{ }^{\circ} \mathrm{C}$ (sample \#GS7).

In contrast to that, the GaSe layer grown at high $\mathrm{T}_{\mathrm{S}} \sim 500{ }^{\circ} \mathrm{C}$ (sample \#GS7) demonstrates strong $\mathrm{PL}$ at $\mathrm{T}=300 \mathrm{~K}$ (Figure $7 \mathrm{~b})$. The spectral position $(1.99 \mathrm{eV})$ of the emission line corresponds well to the emission line of the direct free exciton at $\mathrm{T}=300 \mathrm{~K}$ [74]. The strong anisotropic dependence of the PL intensity (the details can be found in [75]) is evidence of a strictly oriented array of GaSe nanoplatelets in this structure, which is in a good agreement with TEM data.

\subsection{MBE Growth of InSe-Based Heterostructures on GaAs(001) Substrates}

The main problem in the MBE growth of InSe layers is associated with the formation of numerous $\mathrm{In}_{\mathrm{x}} \mathrm{Se}_{\mathrm{y}}$-type phases $[36,76]$. The relation between the film components and $\mathrm{Se} / \mathrm{In}$ flux ratio corresponds to that appearing in the phase diagram [36]. In other words, one can expect the coexistence of $\mathrm{In}_{4} \mathrm{Se}_{3}$ with the main InSe phase at low Se/In flux ratios, as well as the occurrence of In droplets on the growth surface, while at high Se/In flux ratios the $\mathrm{In}_{2} \mathrm{Se}_{3}$ phase will predominantly crystallize. Indeed, the existence of "parasitic" $\operatorname{In}_{x} \mathrm{Se}_{\mathrm{y}}$ phases (mainly $\mathrm{In}_{4} \mathrm{Se}_{3}$ ) has been reported in the InSe layers grown in a wide range of growth conditions on different substrates: glass, $\mathrm{Si}(001), \mathrm{Si}(111), \mathrm{GaAs}(111)$, and GaAs(001) [22,73,77-80]. The most comprehensive study of the film composition as a function of both Se/In flux ratio and $\mathrm{T}_{\mathrm{S}}$ was performed by Emery et al. for the InSe films grown by MBE on amorphous substrates using standard elemental In and Se effusion cells [22,79]. Chatillon et al. explained the experimental results by performing a thorough thermodynamic analysis of MBE growth in the In-Se system [76,81]. The difference between calculated and experimental impinging molecular fluxes was taken into account by introducing the Se incorporation coefficient, which is the usual way for the thermodynamic description of the MBE growth of Se-based semiconductors $[82,83]$. According to Brahim-Otsmane et al. [79], at the substrate temperature of $\mathrm{T}_{\mathrm{S}} \sim 350{ }^{\circ} \mathrm{C}$ for Se/In BEP ratio $\mathrm{R}<2$, which corresponds to the nearly stoichiometric ratio between the adsorbed In and Se 
adatoms on the growth surface [81], mixed films consisting of InSe and $\mathrm{In}_{4} \mathrm{Se}_{3}$ phases are grown, while at $\mathrm{R}>3$ the grown film is composed of $\mathrm{InSe}$ and $\gamma-\mathrm{In}_{2} \mathrm{Se}_{3}$ phases. Moreover, the relatively narrow "MBE growth window" for InSe films $(\mathrm{R}=2-3)$ becomes narrower with increasing growth temperature up to $\mathrm{T}_{\mathrm{S}} \sim 450{ }^{\circ} \mathrm{C}$, and the $\mathrm{In}_{4} \mathrm{Se}_{3}$ phase inclusions are observed even in InSe layers grown at nearly stoichiometric conditions [79,84]. The formation of $\operatorname{In}_{4} \mathrm{Se}_{3}$ phase was also observed during the growth of InSe layers on GaAs(001) substrates, as confirmed by Raman measurements [75].

The growing of InSe films by using a Se valve cracking cell is even more complicated task. It should be noted that $\mathrm{Ga}_{2} \mathrm{Se}_{3}$ phase inclusions were observed in $\mathrm{GaSe} / \mathrm{GaAs}(001)$ layers grown at a high cracking zone temperature $\left(\mathrm{T}_{\mathrm{Se}}(\mathrm{cr})=950^{\circ} \mathrm{C}\right)$ of the Se valve cell even at nearly stoichiometric conditions [30]. In this case, the growth window for "pure" InSe becomes even narrower or, possibly, disappears completely, especially at high $\mathrm{T}_{\mathrm{S}}$. The Se/In BEP ratio corresponding to the nearly stoichiometric conditions is also changed by virtue of a change in the Se sticking coefficient.

In our experiments on MBE growth of InSe/GaAs(001) layers, the standard In cell and Se valve cracking cell with a cracking zone temperature of $\mathrm{T}_{\mathrm{Se}}(\mathrm{cr})=500{ }^{\circ} \mathrm{C}$ were used as molecular beam sources. The In beam flux was $\sim 1.3 \times 10^{-7}$ Torr (BEP), which corresponds to an average InSe growth rate of $1.2-1.3 \mathrm{~nm} / \mathrm{min}$. The layers were grown at $\mathrm{T}_{\mathrm{S}}=450{ }^{\circ} \mathrm{C}$. This temperature was chosen to provide the maximum structural quality of growing film and simultaneously avoid the film re-evaporation. Suggesting the similar dependence of stoichiometric VI/III flux ratio on Se cracking zone temperature as in the case of GaSe, the nearly stoichiometric InSe/GaAs(001) MBE growth conditions at $\mathrm{T}_{\mathrm{S}}=450^{\circ} \mathrm{C}$ were estimated as Se/In(BEP) $\sim 5-6\left(\mathrm{~T}_{\mathrm{Se}}(\mathrm{cr}) \sim 500{ }^{\circ} \mathrm{C}\right)$. The InSe growth on $\mathrm{GaAs}(001)$ was initiated by the simultaneous opening of In and Se shutters.

The plan-view and cross-section SEM images of the InSe film grown directly on GaAs(001) substrate at $\mathrm{T}_{\mathrm{S}}=450{ }^{\circ} \mathrm{C}$ at nearly stoichiometric Se/In flux ratio are presented in Figure 8. One can see the film (hereinafter, sample \#IS1) consists of a number of randomly oriented nanocrystallites, which confirms the difficulties in InSe nucleation on the GaAs(001) surface at high temperatures [73]. The different shape of the nanocrystallites may also indicate the presence of secondary phase inclusions.

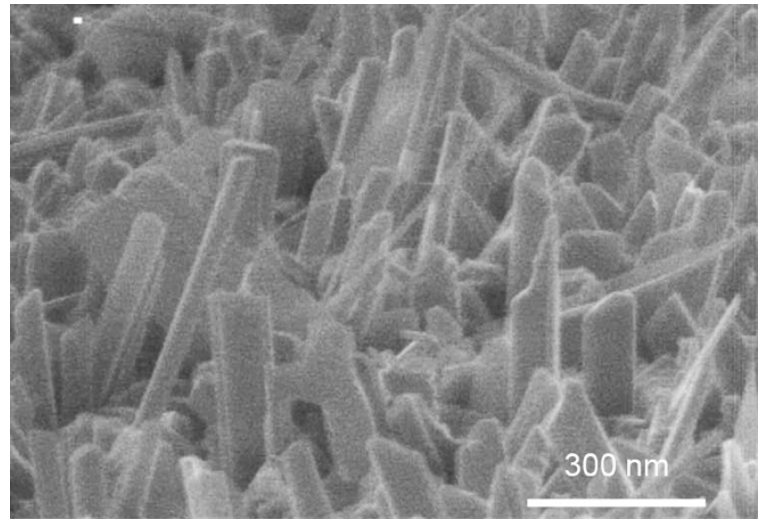

(a)

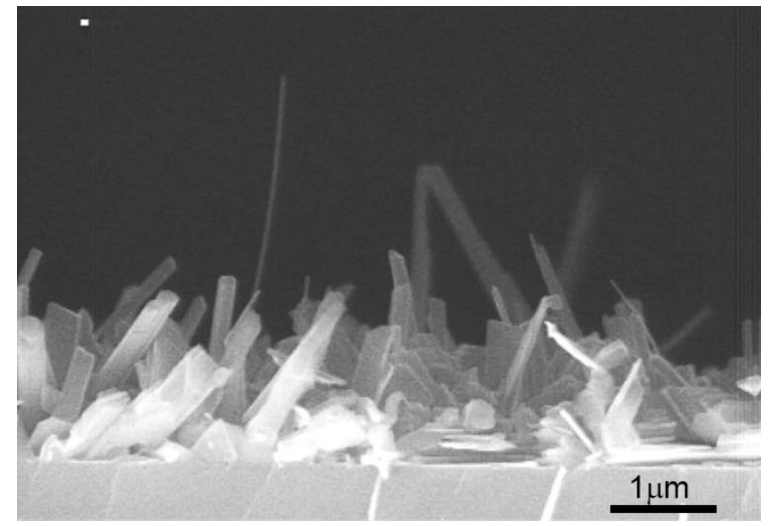

(b)

Figure 8. The plan-view (a) and cross-section (b) SEM images of the InSe/GaAs(001) film (sample \#IS1) grown at $\mathrm{T}_{\mathrm{S}}=450{ }^{\circ} \mathrm{C}$ at nearly stoichiometric growth conditions.

The X-ray powder diffraction pattern of the InSe/GaAs(001) layer (sample \#IS1) is presented in Figure 9. The most intense diffraction peaks are attributed to InSe (001), which indicates that there are a lot of InSe crystallites whose $\mathbf{c}$ axis is perpendicular to the substrate plane. The significant part of additional peaks can be attributed as belonging to the $\mathrm{In}_{4} \mathrm{Se}_{3}$ phase [84,85], and the intensities of these peaks (marked by black circles) are comparable with that from InSe. We assume that the presence of a significant amount of the $\mathrm{In}_{4} \mathrm{Se}_{3}$ phase in the film is associated primarily with the MBE growth conditions used, but also with the use a valve cracking cell as a Se source. Due to the similar positions 
of (003n) and (002n) reflections in the XRD pattern of rhombohedral and hexagonal InSe crystals, it is difficult to conclude which InSe polytype occurs. The intense peak at $2 \theta=29.00$ can be an indication of rhombohedral symmetry, since reflection at this Bragg angle exists only in powder XRD of the rhombohedral InSe modification [86]. However, this peak can also be associated with (040) reflection of $\mathrm{In}_{4} \mathrm{Se}_{3}$. The existence of additional asymmetric reflections on XRD pattern agrees well with observed surface morphology of the film.

The InSe reflections in Figure 9 are indexed for the $\gamma$-InSe rhombohedral lattice, since in this case the positions of asymmetric peaks are found to fit better the measured XRD pattern; we used the lattice parameters of rhombohedral InSe reported by Nagpal and Ali [42]: $\mathrm{a}=4.0046 \AA$ and $\mathrm{c}=24.960 \AA$.

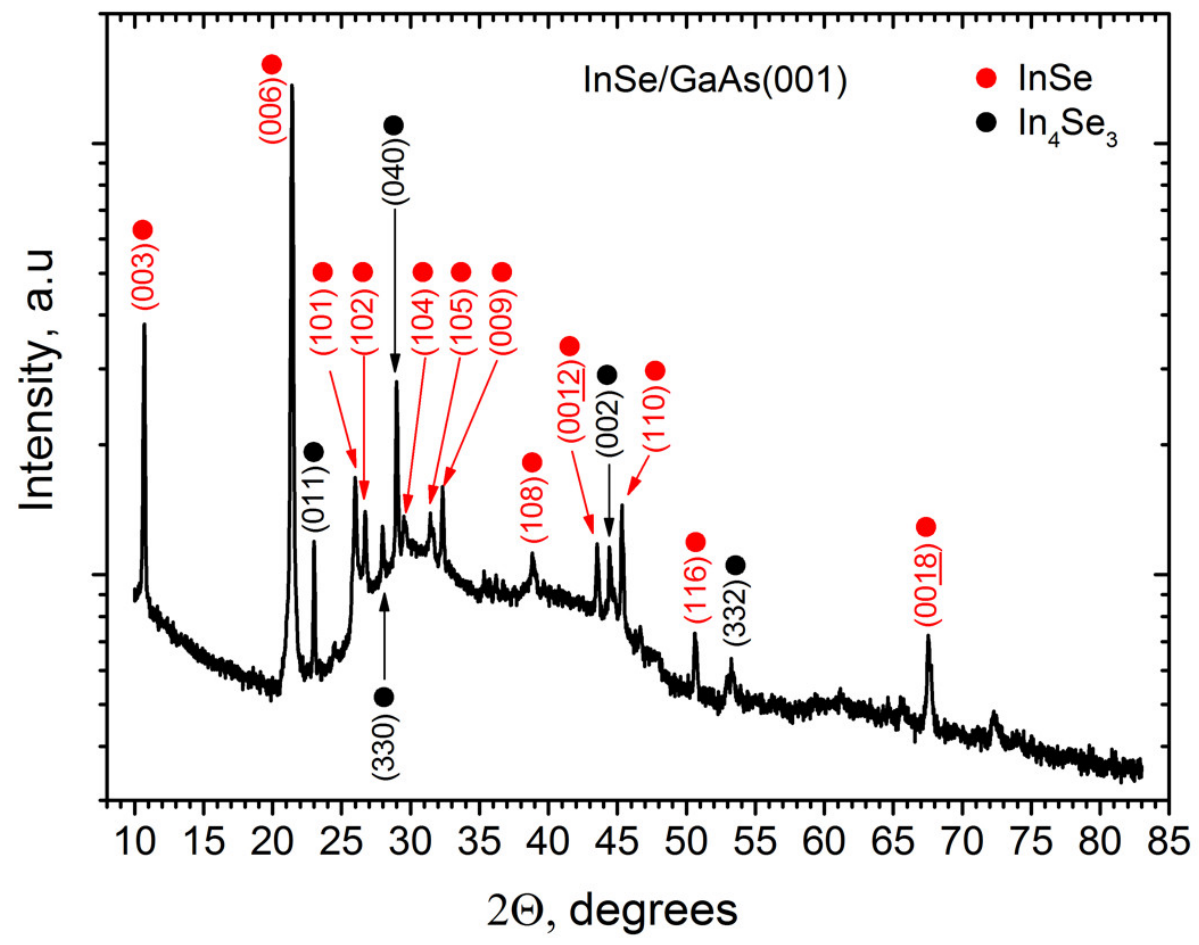

Figure 9. The X-ray powder diffraction pattern of the InSe/GaAs(001) layer (sample \#IS1). Reflections corresponding to InSe are indicated by red circles, while reflections that can be attributed to the $\mathrm{In}_{4} \mathrm{Se}_{3}$ phase are marked by black circles. The InSe reflections are indexed for the $\gamma$-InSe rhombohedral lattice.

Raman spectra of the InSe/GaAs(001) layer (sample \#IS1) measured at 80 and $300 \mathrm{~K}$ are shown in Figure 10. The line at $532 \mathrm{~nm}$ of Nd:YAG laser was used as the excitation source. Five peaks located at 18, $41,115,177$, and $227 \mathrm{~cm}^{-1}$ are clearly seen in the room temperature Raman spectrum. These values are typical for InSe phonon modes measured under off-resonance conditions [87]. The phonon frequencies increase by a few $\mathrm{cm}^{-1}$ with decreasing temperature from 300 to $80 \mathrm{~K}$. Additional peaks in the low temperature Raman spectrum detected at $38,72,104$, and $168 \mathrm{~cm}^{-1}$ can be associated with the presence of $\mathrm{In}_{4} \mathrm{Se}_{3}$ phase $[73,84]$, which is in a good agreement with XRD measurements. As mentioned above, according to XRD data, the InSe/GaAs(001) layer has a rhombohedral lattice, which indicates a $\gamma$-InSe polytype. However, the presence in the Raman spectrum of a low-frequency line at $18 \mathrm{~cm}^{-1}$ is in contradiction with this statement. According to the group-theory analysis, the lowest frequency line in the Raman scattering spectrum of InSe should correspond to interlayer vibrations [88]. The primitive unit cell of $\gamma$-InSe contains only one layer per unit cell and therefore this line should not be observed in the first-order Raman spectra. The appearance of this line is an important signature of presence of either $\varepsilon$ - or $\beta$-phase InSe [87]. On the other hand, in the Raman spectra of non-centrosymmetric $\gamma$ - and $\varepsilon$-InSe polytypes, polar longitudinal (LO) modes should be observed in certain scattering geometries and the intensity of those modes is enhanced in resonance conditions [89-91]. 


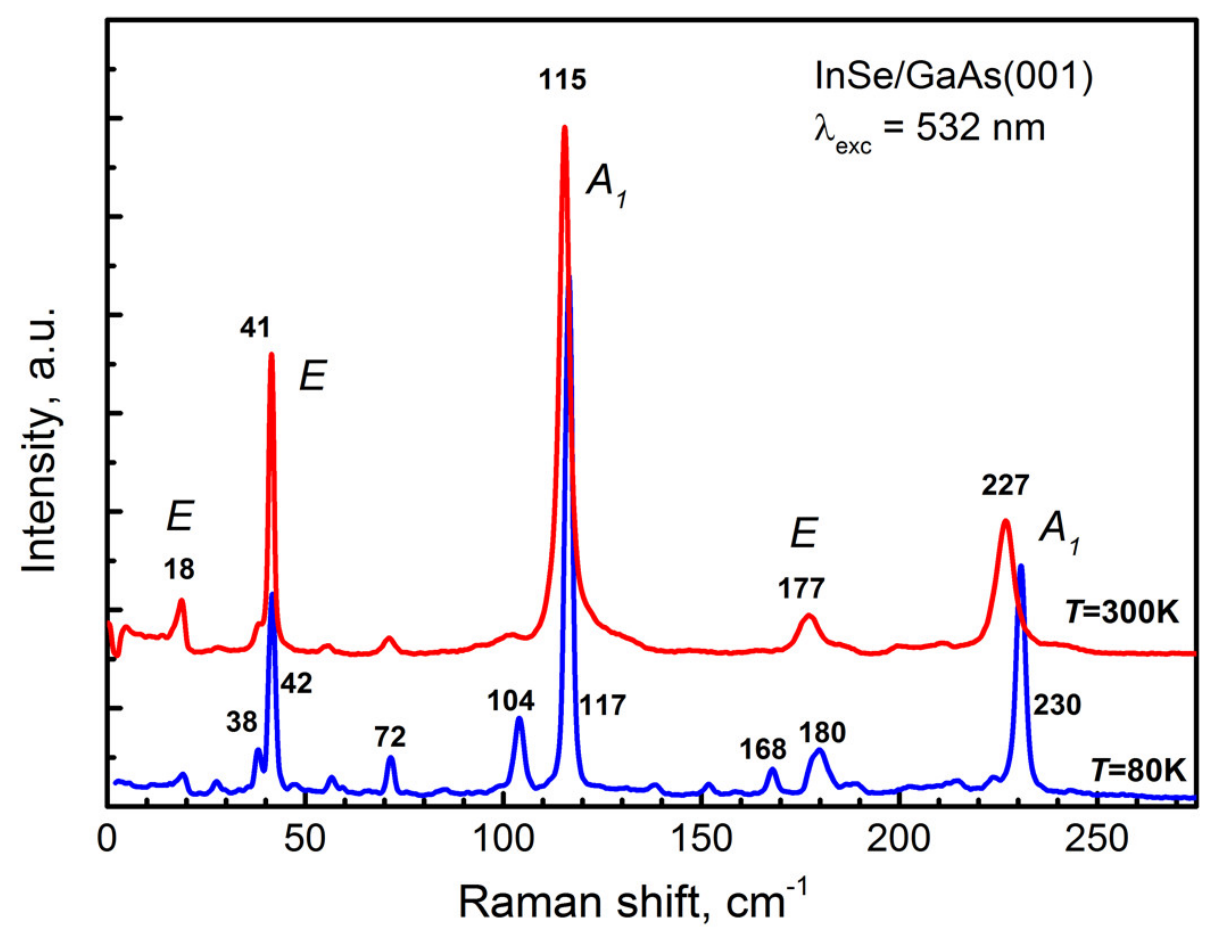

Figure 10. The Raman spectra of the InSe/GaAs(001) layer (sample \#IS1) measured at $300 \mathrm{~K}$ (red curve) and at $80 \mathrm{~K}$ (blue curve). The symmetrycep of the phonon modes are given in accordance with [87].

To clarify the reason for the contradiction between the XRD and Raman data, the measurements were made in the near-resonance Raman scattering using 514.5 and $488 \mathrm{~nm}$ lines of Argon laser as excitation sources. For $\gamma$-InSe, the $514.5 \mathrm{~nm}$ line has energy of about $10 \mathrm{meV}$ below the $\mathrm{E}_{1}^{\prime}$ exciton energy, while the energy of the 488 line is $9 \mathrm{meV}$ above the exciton energy at the respective temperatures [91]. The corresponding spectra are shown in Figure 11 together with the off-resonance Raman spectrum obtained using the line at $532 \mathrm{~nm}$ as an excitation source. It can be seen that at room temperature a new doublet feature with frequencies of $199 \mathrm{~cm}^{-1}$ and $211 \mathrm{~cm}^{-1}$ is observed upon excitation at $514.5 \mathrm{~nm}$. In the spectrum at $\mathrm{T}=80 \mathrm{~K}$ obtained upon $488 \mathrm{~nm}$ excitation, the doublet feature experiences a high-frequency shift and a significant increase in intensity. Similar spectra for $\gamma$-rich bulk InSe were reported for the first time by Kuroda and Nishina [91]. It should be noted that in this work, upon excitation by the 514.5 and $488 \mathrm{~nm}$ lines, a low-frequency line was detected in the region of about $17-18 \mathrm{~cm}^{-1}$ in the Raman spectra (similar to that shown in Figure 11). However, this line, as already discussed above, should not be observed for the pure $\gamma$-InSe.

The spectra shown in Figure 11 make it possible to exclude the presence of $\beta$-phase in the InSe/GaAs(001) layer, since polar (LO) phonon modes that could be resonantly enhanced are absent in the Raman spectrum of $\beta$-InSe polytype [90]. Thus, taking into account the XRD data, the InSe/GaAs(001) layer (sample \#IS1) can be characterized as $\gamma$-rich InSe, implying that along with the main $\gamma$-phase of InSe there exists also a certain amount of $\varepsilon$-phase. This result is in good agreement with the fact that MBE grown InSe films are usually of $\gamma$-polytype $[73,78,80,92,93]$.

The PL spectra of the InSe/GaAs(001) layer (sample \#IS1) measured within the temperature range of $11-110 \mathrm{~K}$ are presented in Figure 12. A $405 \mathrm{~nm}$ violet diode laser SSP-DHS-405 was used as the excitation source; the excitation power was as low as $50 \mathrm{~mW}$, which corresponds to the excitation power density of $\sim 0.5 \mathrm{~W} / \mathrm{cm}^{2}$. The highest-energy peak observed in the PL spectrum at $\mathrm{T}=11 \mathrm{~K}$ is detected at $\sim 1.307 \mathrm{eV}$. This is more than $30 \mathrm{meV}$ lower than the exciton recombination line $(1.338 \mathrm{eV})$ recently reported by Shubina et al. [94] for flakes freshly cleaved from high structural quality bulk InSe grown by the Bridgman-Stockbarger method. According to the previous results [95,96], in the region below $1.32 \mathrm{eV}$, three main broad bands around 1.31,1.28, and $1.23 \mathrm{eV}$ have been reported in undoped InSe, which were assigned to impurity-band, donor-acceptor pair, and impurity-vacancy complex 
transitions, respectively. In a good agreement with these reports, the low-temperature spectrum shown in Figure 12 (at $\mathrm{T}=11 \mathrm{~K}$ ) can be also approximated by at least three different Gaussian peaks centered at $1.307,1.28$, and $1.23 \mathrm{eV}$, respectively. The broad peak at $1.307 \mathrm{eV}(\mathrm{T}=11 \mathrm{~K})$, which dominates the spectrum at low temperatures, quenches quickly with increasing temperature and disappears at $\mathrm{T} \approx 50 \mathrm{~K}$. At temperatures higher than $40 \mathrm{~K}$, the defect-related band begins to dominate in the spectra. However, at temperatures higher than $60 \mathrm{~K}$, one can see an occurrence of a clearly resolved peak centered at $\sim 1.314 \mathrm{eV}$. From the PL temperature dependence, we can assume that the initial broad peak centered at $\sim 1.307 \mathrm{eV}(\mathrm{T}=11 \mathrm{~K}$ ) actually consists of two closely spaced peaks located at 1.301 and $1.315 \mathrm{eV}$. Indeed, the spectrum at $\mathrm{T}=11 \mathrm{~K}$ is most accurately approximated by four Gaussian contours (Figure S6). Homs and Marí [97] performed a comparative study of the PL of undoped and slightly neutron-transmutation doped InSe samples. In the region around $1.31 \mathrm{eV}$ in doped samples, they found two closely-spaced and poorly separated bands, which they indexed as $E$ and $F$, respectively. These bands correspond well to $P 1$ and $P 2$ peaks in Figure S6. They found also that emission $F$ thermally quenches faster than $E$. The peak $E$ was attributed to the recombination of a native acceptor, while peak $F$ was assigned to donor-acceptor pair transition where both donors and acceptors are ionized. Indeed, in our case, we observe a similar temperature dependence of P1 and P2 peaks (see Figure 12a) and, accordingly, we can accept the proposed interpretation. In the same way, the band at $1.28 \mathrm{eV}$ also can be attributed to a donor-acceptor pair transition [96,97] due to its fast temperature quenching. Thus, these three transitions $(P 1, P 2$, and $P 3)$ could be assigned to InSe native impurities (defects) because they have been observed in undoped samples [97]. Finally, the broad band observed at $\sim 1.23 \mathrm{eV}$ at $\mathrm{T}=11 \mathrm{~K}$ can be associated with structural defects in the crystal. The presence of this band is an indication of a high concentration of defects and this band is assigned to a transition within an impurity vacancy-complex $[95,96]$.

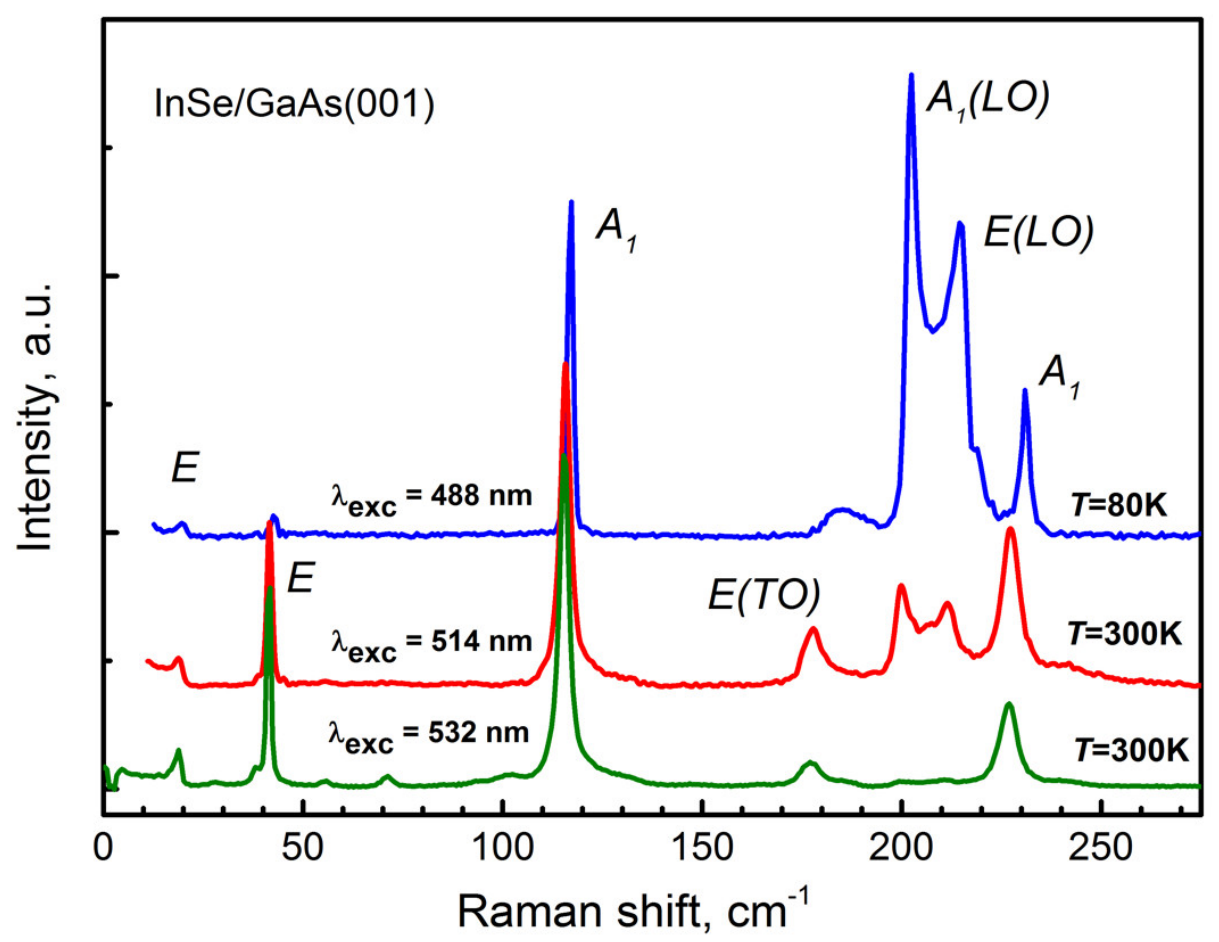

Figure 11. The near-resonance Raman spectra of the InSe/GaAs(001) layer (sample \#IS1) measured at $300 \mathrm{~K}\left(\lambda_{\text {exc }}=514.5 \mathrm{~nm}\right.$, red curve $)$ and at $80 \mathrm{~K}\left(\lambda_{\text {exc }}=488 \mathrm{~nm}\right.$, blue curve $)$ along with the off-resonance Raman spectrum of the same layer measured at $300 \mathrm{~K}\left(\lambda_{\mathrm{exc}}=532 \mathrm{~nm}\right.$, green curve $)$. The spectra are normalized to the intensity of the $\mathrm{A}_{1}\left(115 \mathrm{~cm}^{-1}\right)$ phonon line. 

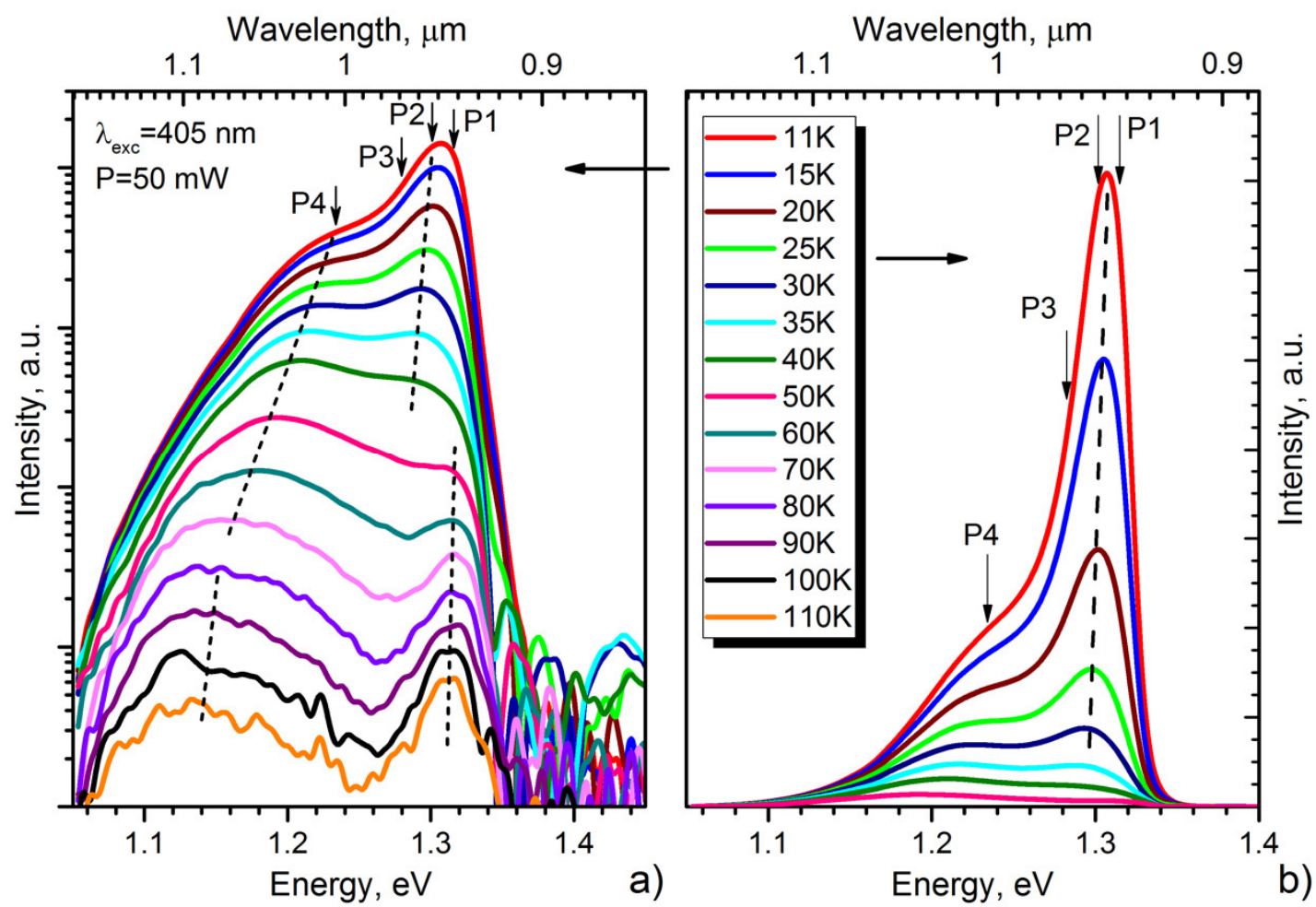

Figure 12. Photoluminescence spectra of the InSe/GaAs(001) layer (sample \#IS1) at a low excitation power of $0.5 \mathrm{~W} / \mathrm{cm}^{2}$ in logarithmic (a) and linear (b) scales measured at different temperatures. The colors of the curves correspond to the legend presented in (b). The P1, P2, P3 and P4 peaks correspond to those shown in Figure S6. The dashed lines are shown for eyes only and correspond to temperature shift of the respective peak maxima.

To improve the surface morphology of the layer, it is apparently possible to use the two-stage growth technique, which involves the deposition of the InSe buffer layer at low $\mathrm{T}_{\mathrm{S}}$ in order to form a more planar interface $[98,99]$. A more advanced strategy involves the use of a GaSe buffer layer $[73,80]$. To control the film orientation, Budiman et al. also used the $\mathrm{GaAs}(001)$ substrates misoriented by $2^{\circ}$ or $5^{\circ}$ toward [110] direction [73]. However, despite the relatively planar surface morphology of the GaSe buffer layer (see Section 3.1), the top InSe layer grown at high $\mathrm{T}_{\mathrm{S}} \sim 450{ }^{\circ} \mathrm{C}$ is again composed of a number of microcrystallites (see Figure S7). The observation of Debye rings in the RHEED pattern during MBE growth of InSe also confirms the polycrystalline nature of the film.

The strong diffusion of Ga into the top InSe layer with the formation of the InGaSe alloy was observed in the InSe/GaSe/GaAs(001) heterostructure, where the InSe growth temperature was as high as $\mathrm{T}_{\mathrm{S}} \approx 465^{\circ} \mathrm{C}$. The RT Raman spectrum of this structure is presented in Figure 13 (upper curve) along with the Raman spectra of reference InSe/GaAs(001) (middle curve) and $\gamma$-GaSe/GaAs(001) (lower curve) layers. The Ga diffusion into the top InSe layer is confirmed by the shift of all InSe Raman peaks in comparison with the reference InSe/GaAs(001) layer. The weak peaks at 59 and $132 \mathrm{~cm}^{-1}$ in the upper curve in Figure 13 correspond to the underlying GaSe buffer layer. It should be noted that strong interdiffusion at the InSe/GaSe interface at the growth temperatures of InSe higher than $400{ }^{\circ} \mathrm{C}$ was observed also in previous works $[73,80]$.

Taking account of difficulties in InSe/GaAs(001) heteroepitaxy, as well as the problems with high-temperature InSe growth on GaSe surface, we tried to realize the ZnSe/InSe/ZnSe quantum well $(\mathrm{QW})$ heterostructures. The InSe/ZnSe looks as a promising heteropair for potential applications in the field of photonics and electronics, when taking into account the fact that the band line-up for the InSe/ZnSe interface was reported to be of strong type I [100] as well as the existence of matured MBE technology of $\mathrm{ZnSe} / \mathrm{GaAs}(001)$ [59]. 


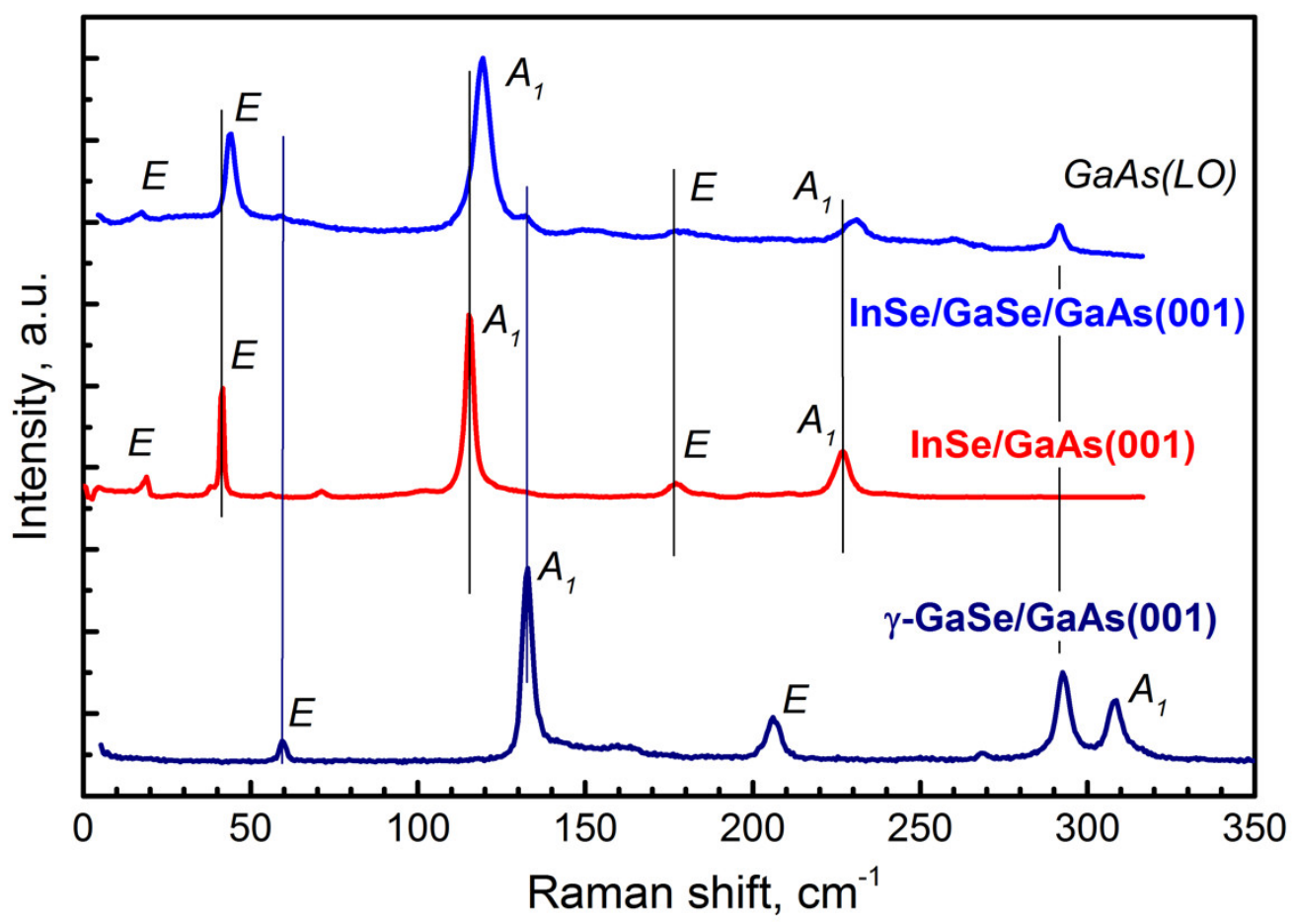

Figure 13. The Raman spectrum (upper curve) of the InSe/GaSe/GaAs(001) structure along with the Raman spectra of both InSe/GaAs(001) (sample \#IS1, middle curve) and $\gamma$-GaSe/GaAs(001) (sample \#GS1, lower curve) layers $(T=300 \mathrm{~K})$. The spectra are normalized to the intensity of the $\mathrm{A}_{1}\left(115 \mathrm{~cm}^{-1}\right)$ phonon line.

The nominal thickness of InSe layer in the ZnSe/InSe/ZnSe QW heterostructures ranged 2-7 TLs. The thicknesses of the ZnSe bottom and top layers were 80-160 and 20-40 nm, respectively. The growth conditions of InSe on $\mathrm{ZnSe}(001)$ surface were chosen the same as in the case of InSe/GaSe heteroepitaxy: $\mathrm{T}_{\mathrm{S}}=450^{\circ} \mathrm{C}$ and flux ratio Se/In (BEP) 5-6. The top ZnSe layer on the InSe surface was grown at $\mathrm{T}_{\mathrm{S}}=300^{\circ} \mathrm{C}$. The details of MBE growth of ZnSe/InSe/ZnSe QW heterostructures as well as studies of their surface morphology were recently reported by Avdienko et al. [101]. Here, we focus mainly on the structural and optical properties of these QW structures.

Figure 14a shows the cross-sectional high-resolution TEM image of the ZnSe/InSe/ZnSe QW structure with a 7-TL-thick InSe layer. The TEM data confirm the multi-domain character of InSe/ZnSe(001) nucleation. At the growth conditions used, the $c$ axis of InSe is directed normal to the growth surface. The TEM data also confirm the (111)-oriented ZnSe growth on the InSe(0001) surface, as previously reported by Smathers et al. [102]. However, the orientation of the ZnSe layer remains unchanged in those parts of the structure where there are no InSe islands. The existence of the (111)-oriented ZnSe phase in the structure was also confirmed by SAED measurements [101]. We suppose that the growth of ZnSe with a (111) orientation onto the InSe(0001) van der Waals surface is caused by the proximity of the InSe and $\mathrm{ZnSe}(111)$ lattice parameters $(\Delta \mathrm{a} / \mathrm{a} \sim 1 \%$ or less, $\mathrm{a}(\mathrm{InSe})=4.005 \AA$ [41]). Therefore, the InSe islands provide the epitaxial orientation of the upper ZnSe layer, despite the weak interaction between them. In this case, the island-like growth of ZnSe(111) can be explained in terms of minimizing the elastic energy of the growing layer [101].

All QW structures demonstrate similar PL independent from InSe nominal thickness. One can see the intense broad line centered at $\sim 2.27 \mathrm{eV}$ (Figure $14 \mathrm{~b}$ ), indicating the same origin of this line in all QW structures. The time-resolved PL measurements demonstrate that the PL decay time is large and also nearly the same ( $270 \mathrm{~ns})$ in all structures (Figure 14c). Therefore, we can assume the luminescence is associated with radiative recombination centers of In in ZnSe because of the In diffusion into the underlying ZnSe layer. This assumption agrees well with the experimental value of the acceptor level 
energy in ZnSe crystals doped with indium, which is $\sim 0.41 \mathrm{eV}$ above the valence band [103]. A similar PL line at $\sim 2.25 \mathrm{eV}$ was also previously observed in In-doped ZnSe crystals [104].
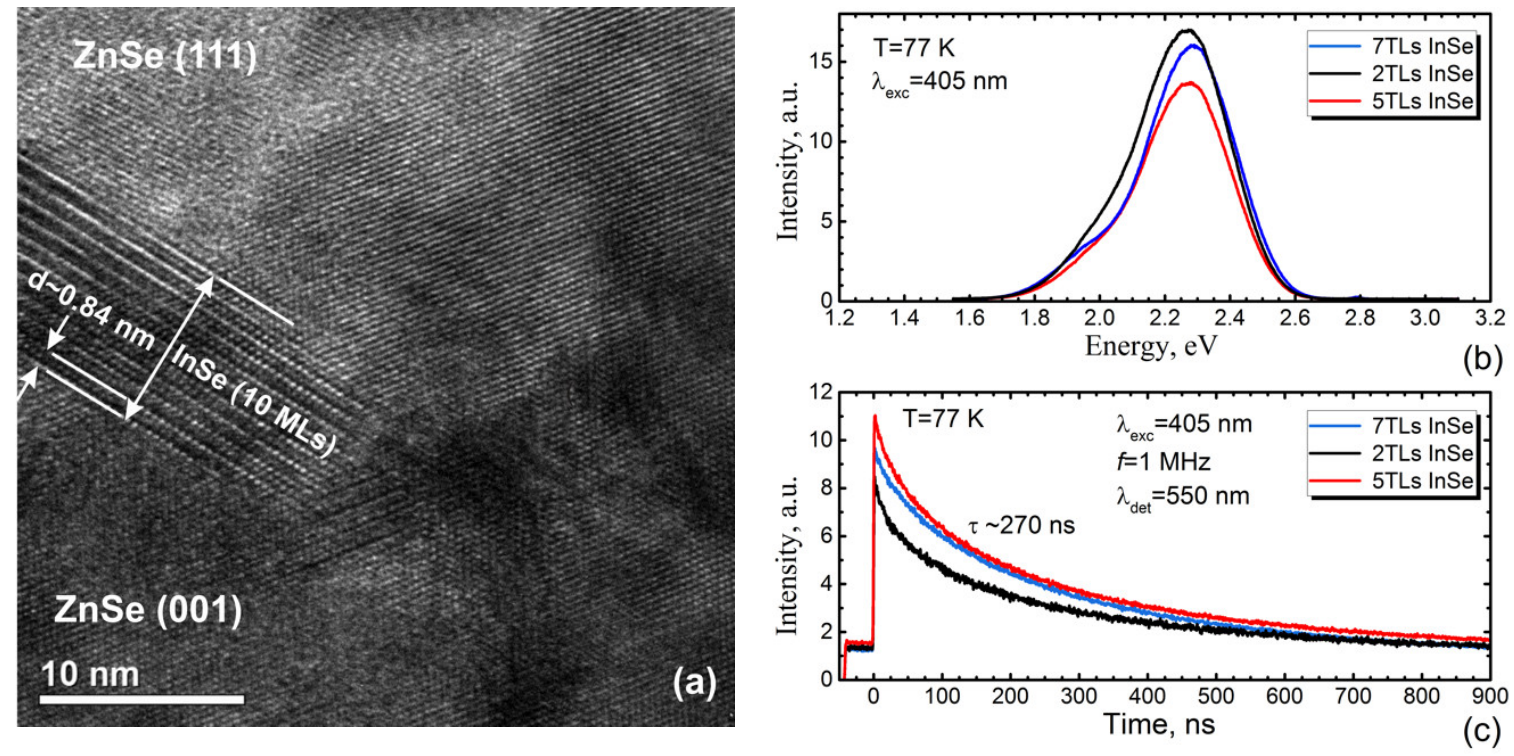

Figure 14. (a) Cross-section HRTEM image of the ZnSe/InSe/ZnSe QW structure with the InSe nominal thickness of 7 TLs; (b) PL spectra of ZnSe/InSe/ZnSe QW structures with different InSe nominal thickness; (c) time-resolved measurements of the luminescence in $\mathrm{ZnSe} / \mathrm{InSe} / \mathrm{ZnSe} \mathrm{QW}$ structures at $\mathrm{T}=77 \mathrm{~K}$. The samples were excited by $405 \mathrm{~nm}$ radiation of the CUBE laser (Coherent) with pulses of 45 ps duration at a repetition rate of $1 \mathrm{MHz}$.

\subsection{MBE Growth of GaTe Layers and GaTe/GaSe Heterostructures on GaAs(001) Substrates}

As mentioned above, one of the main problem in the MBE growth of different layered III-VI's is the formation of numerous "parasitic" phases, i.e., $\operatorname{In}_{4} \mathrm{Se}_{3}$ and $\operatorname{In}_{2} \mathrm{Se}_{3}[73,101]$ or $\mathrm{Ga}_{2} \mathrm{Se}_{3}[28,30]$ in the case of MBE growth of InSe and GaSe, respectively. For the Ga-Te system, the other possible stable phase is $\mathrm{Ga}_{2} \mathrm{Te}_{3}$ [105]. The $\mathrm{Ga}_{2} \mathrm{Te}_{3}$ films were successfully synthesized by metalorganic MBE on both $\operatorname{InP}(001)$ and $\mathrm{GaAs}(001)$ substrates at $\mathrm{T}_{\mathrm{S}}=400-500{ }^{\circ} \mathrm{C}$ under strong Te-rich conditions $(\mathrm{Te} / \mathrm{Ga} \sim 30)[106,107]$. However, no evidence of $\mathrm{Ga}_{2} \mathrm{Te}_{3}$ inclusions has been obtained in $\mathrm{MBE}$ grown $\mathrm{GaTe} / \mathrm{GaAs}(001)$ epilayers at much lower $(\mathrm{Te} / \mathrm{Ga} \sim 10)$ flux ratio [108].

In this study, GaTe layers were grown on $\mathrm{GaAs}(001)$ substrates via a 200-nm-thick GaAs buffer layer under Te-rich conditions at low $\mathrm{T}_{\mathrm{S}} \approx 450{ }^{\circ} \mathrm{C}$ (sample \#GT1) and high $\mathrm{T}_{\mathrm{S}} \approx 530{ }^{\circ} \mathrm{C}$ (sample \#GT2) substrate temperatures, respectively. The GaTe growth was initiated by the simultaneous opening of both Te and Ga fluxes onto the GaAs(001) surface. The Te/Ga flux ratios were chosen to provide the Te-rich conditions in accordance with Bae et al. [108]. The growth parameters as well as thicknesses of GaSe layers are outlined in Table 2.

Table 2. The growth parameters of GaTe/GaAs (001) layers.

\begin{tabular}{|c|c|c|c|c|c|c|}
\hline Layer & $\begin{array}{l}\mathrm{T}_{\mathrm{Ga} ; \mathrm{Ga} \text { Flux }} \\
\text { (BEP) }\end{array}$ & $\begin{array}{c}\text { Te/Ga Flux Ratio, } \\
\text { (BEP) }\end{array}$ & $T_{\mathrm{S}},{ }^{\circ} \mathrm{C}$ & $\begin{array}{l}\text { Layer Thickness, } \\
\text { nm (SEM) }\end{array}$ & $\begin{array}{l}\text { Average Growth } \\
\text { Rate }^{2}, \mathrm{~nm} / \mathrm{min}\end{array}$ & Notes \\
\hline \#GT1 & $\begin{array}{c}900^{\circ} \mathrm{C} ; \\
4.0 \times 10^{-8} \text { Torr. }\end{array}$ & $\mathrm{Te} / \mathrm{Ga}(\mathrm{BEP}) \sim 11$ & 450 & 230 & 1.28 & $\begin{array}{c}0.2-\mu \mathrm{m} \text {-thick GaAs } \\
\text { buffer layer }\end{array}$ \\
\hline \#GT2 & $\begin{array}{c}920^{\circ} \mathrm{C} \\
6 \times 10^{-8} \text { Torr. }\end{array}$ & $\mathrm{Te} / \mathrm{Ga}(\mathrm{BEP}) \sim 15$ & 530 & 200 & 1.82 & $\begin{array}{c}0.2-\mu \mathrm{m} \text {-thick GaAs } \\
\text { buffer layer }\end{array}$ \\
\hline
\end{tabular}

As mentioned above, GaTe preferentially crystallizes in the monoclinic $\alpha$-structure (m-GaTe), which is more stable than the hexagonal close-packed $\beta$-structure (h-GaTe) [108-110]. The metastable phase h-GaTe generally exists in ultrathin samples $[109,111]$. However, under appropriate growth 
conditions, thick hexagonal GaTe flakes can be also obtained. Yu et al. recently demonstrated the phase-controlled synthesis of monoclinic and hexagonal GaTe using conventional physical vapor deposition technique [112]. They found that growth temperature is the key factor for phase control of GaTe flakes, and h-GaTe flakes are intended to be stable under relatively low deposition temperature. The importance of low deposition temperature for h-GaTe fabrication is also in a good agreement with the growth conditions reported for the GaTe films grown by metal-organic chemical vapor deposition [113]. According to reported results, h-GaTe is a thermodynamically unstable phase and converts into monoclinic one (m-GaTe) with the increase in sample thickness or environmental temperature $[108,109,113]$. In particular, the thickness-dependent transformation of h-GaTe to m-GaTe has been reported for the GaTe layers grown by MBE on GaAs(001) substrates [108]. At substrate temperature $\mathrm{T}_{\mathrm{S}} \approx 450{ }^{\circ} \mathrm{C}$ and $\mathrm{Te} / \mathrm{Ga}(\mathrm{BEP})=10$, the critical thickness of such transformation has been estimated as high as $\sim 90 \mathrm{~nm}$.

The growth temperature is the main factor affecting the properties of the III-VI layers. Generally, the layers grown at higher $T_{S}$ demonstrate the better crystal and optical quality. The plan-view SEM images of the samples \#GT1 and \#GT2 grown at different $T_{S}$ are presented in Figure 15. The surface morphology of sample \#GT1 (Figure 15a) is typical for the MBE grown III-VI layers and is very similar to the surface morphology observed in the GaSe layers grown at low $\mathrm{T}_{\mathrm{S}} \sim 400{ }^{\circ} \mathrm{C}$ (see Figure 2). In contrast, the plan-view SEM image of sample \#GT2 (Figure 15b) exhibits pronounced surface relief anisotropy, which is either related to the strong anisotropic nature of the m-GaTe crystal structure or induced by the large lattice mismatch between the GaTe and GaAs(001) substrate.
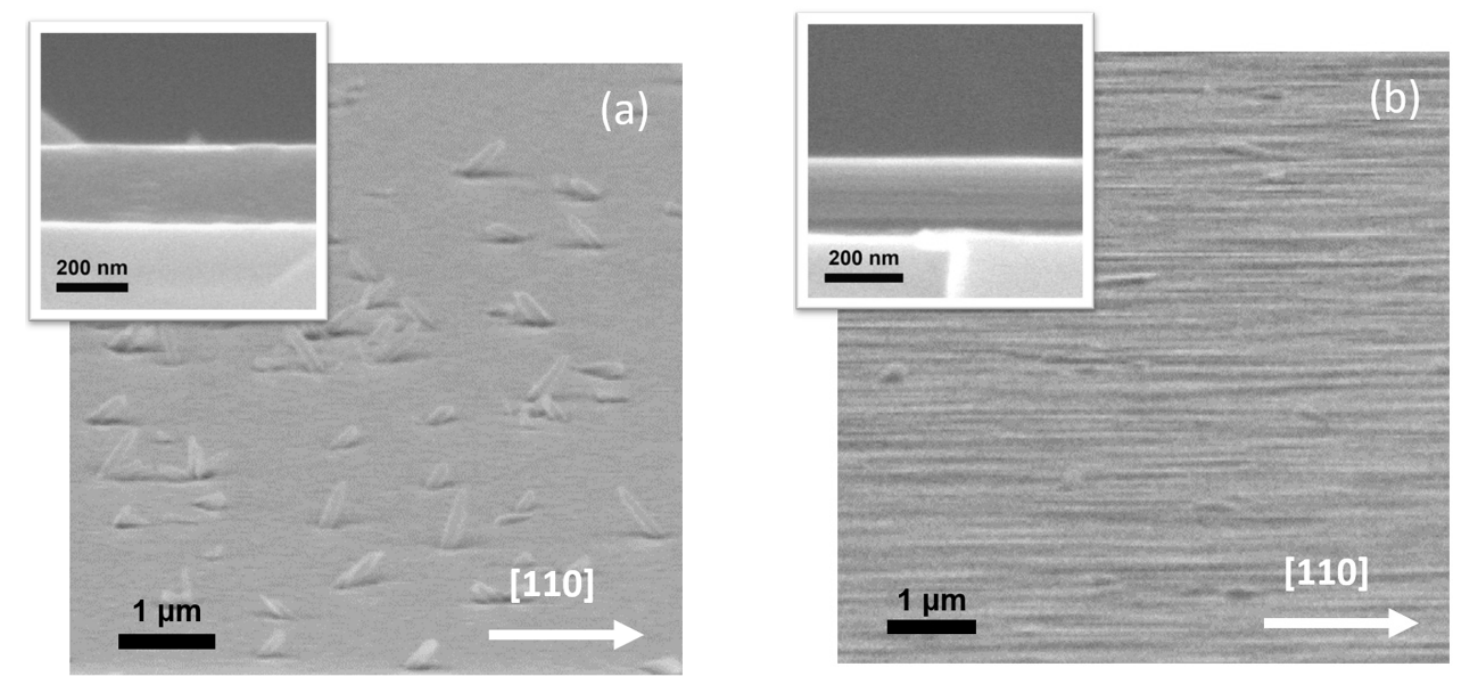

Figure 15. Plan-view SEM images of the GaTe layers grown on GaAs(001) substrates at different substrate temperature: (a) sample \#GT1, $\mathrm{T}_{\mathrm{S}} \approx 450{ }^{\circ} \mathrm{C}$; (b) sample \#GT2, $\mathrm{T}_{\mathrm{S}} \approx 530{ }^{\circ} \mathrm{C}$. The insets show the cross-section SEM images of corresponding structures.

An increase in the growth temperature can lead to chemical interaction between the substrate and the growing layer. This interaction in case of GaSe/GaAs(001) MBE growth $\left(\mathrm{T}_{S}>500{ }^{\circ} \mathrm{C}\right)$ results in the significant surface morphology transformation because of the growth of inclined nanoplatelets (see Figure 4). However, the cross-section SEM image of GaTe layer (sample \#GT2) (see inset in the Figure $15 \mathrm{~b}$ ) grown at high $\mathrm{T}_{\mathrm{S}} \approx 530^{\circ} \mathrm{C}$ demonstrates the formation of a relatively flat layer with nearly abrupt $\mathrm{GaTe} / \mathrm{GaAs}(001)$ interface.

The RHEED images during the growth of samples \#GT1 and \#GT2 are presented in Figure 16. One can see that the RHEED patters remain streaky for both samples. However, the reflections for sample \#GT1 grown at low temperature $\left(T_{S} \approx 450{ }^{\circ} \mathrm{C}\right.$ ) are thickened and blurred (Figure 16a,b), and the brightness of the RHEED pattern gradually decreased with increasing the layer thickness. This behavior reflects an increase in the surface roughness as the layer grows, and can be induced by the formation 
of multi-domain structure at the initial growth stage, i.e., the MBE growth of GaTe is similar to that observed for $\mathrm{GaSe} / \mathrm{GaAs}(001)$ at low substrate temperatures. In contrast, the RHEED pattern of sample \#GT2 $\left(\mathrm{T}_{\mathrm{S}} \approx 530^{\circ} \mathrm{C}\right.$ ) demonstrates much narrower streaky reflections (Figure 16c), confirming that the higher is the growth temperature, the higher is the crystal quality of the growing film.
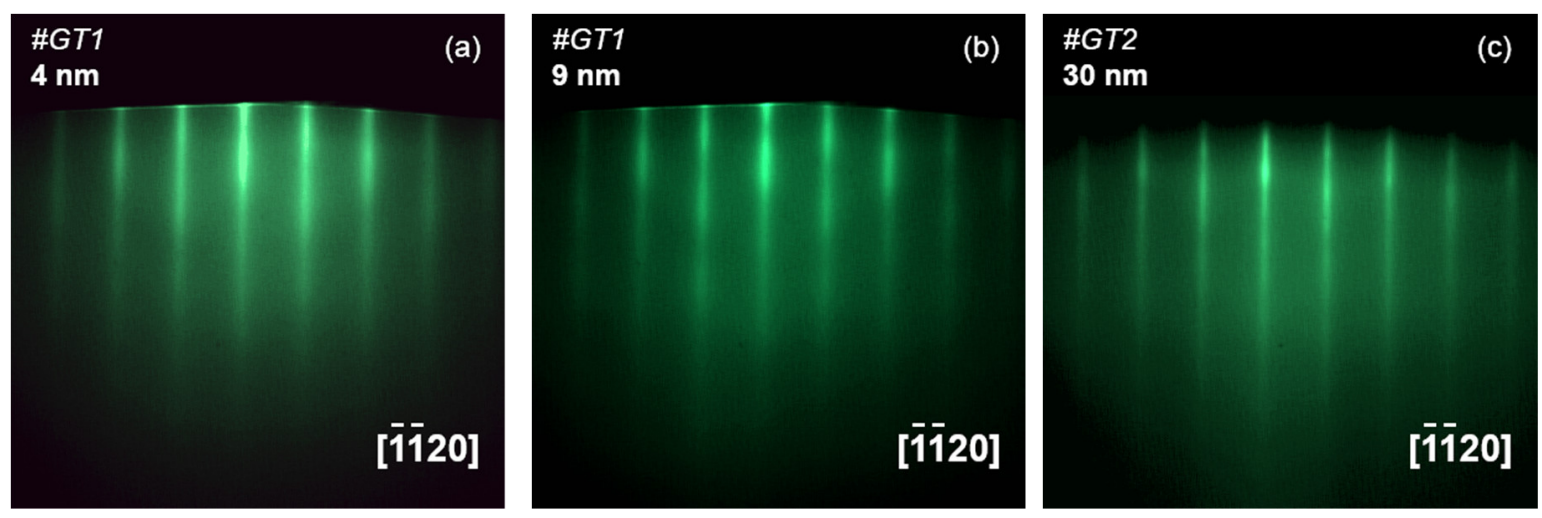

Figure 16. The evolution of the RHEED patterns along the [1120] direction in MBE of GaTe/GaAs(001) epilayers grown at low $\mathrm{T}_{\mathrm{S}} \approx 450{ }^{\circ} \mathrm{C}$ (sample \#GT1): (a) the layer thickness of $\sim 4 \mathrm{~nm}$; (b) the layer thickness of $\sim 9 \mathrm{~nm}$; (c) the RHEED pattern of sample \#GT2 grown at $\mathrm{T}_{\mathrm{S}} \approx 530{ }^{\circ} \mathrm{C}$ (the layer thickness is $\sim 30 \mathrm{~nm})$.

To understand the difference between samples \#GT1 and \#GT2, we performed PL measurements. The as-grown samples were transferred through the air from MBE load-lock chamber into a closed-cycle helium cryostat. After that, they were stored in vacuum at room temperature for three weeks. The PL spectra were measured at $\mathrm{T}=11 \mathrm{~K}$ in a closed-cycle helium cryostat using a laser source with the excitation wavelength of $\lambda_{\text {exc }}=405 \mathrm{~nm}$. The laser power on the sample was as low as $50 \mathrm{~mW}$ with a spot of $\sim 0.1 \mathrm{~cm}^{2}$, which corresponds to the excitation power density of $0.5 \mathrm{~W} / \mathrm{cm}^{2}$. PL spectra of sample \#GT2, measured after different storage times (within three weeks) are shown in Figure 17. The spectra are almost identical, confirming the absence of degradation of the GaTe optical properties during vacuum storage.

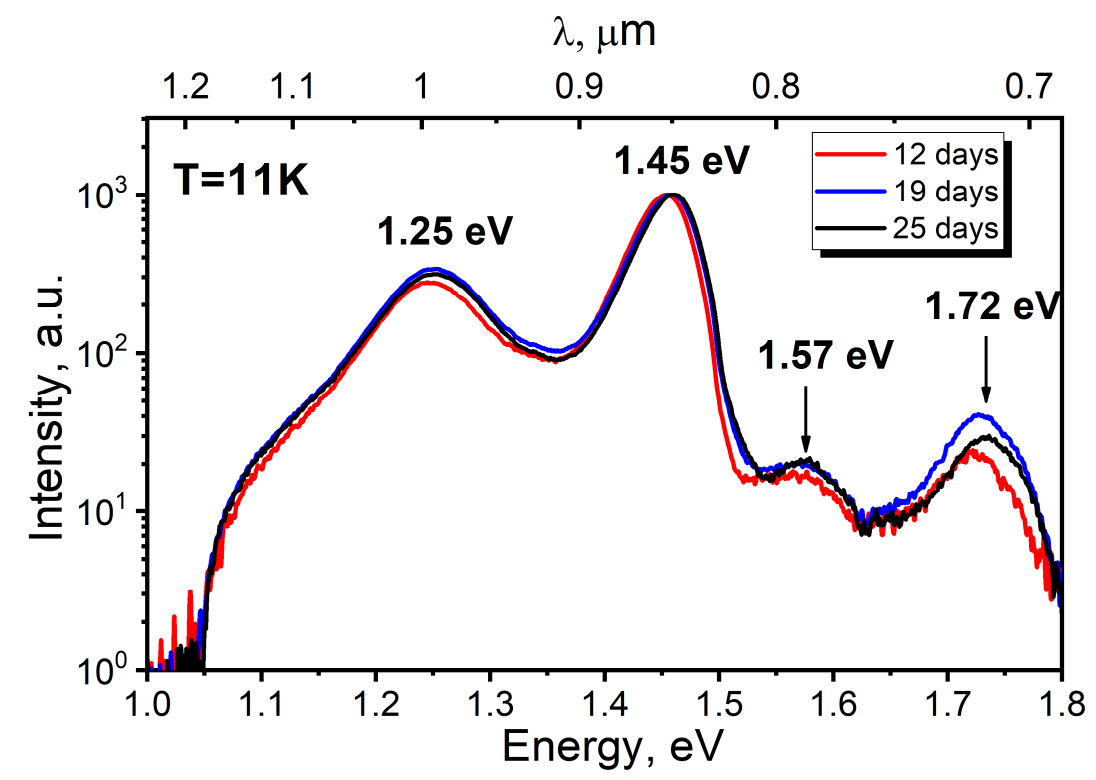

Figure 17. PL spectra of the GaTe/GaAs(001) layer \#GT2 measured after different storage time in vacuum (within three weeks). The PL intensity is normalized to the PL peak with energy of $1.45 \mathrm{eV}$. 
The shortest wavelength peak located at $\sim 1.72 \mathrm{eV}$ could be associated with the emission of acceptor-bound excitons [114], while the broad band centered at $1.57 \mathrm{eV}$ is presumably associated with donor-acceptor pair (DAP) recombination [115]. This interpretation is in good agreement with the assumption that Te-rich growth conditions should cause the GaTe layer to exhibit p-type natural conductivity [108]. One of the possible explanations of $1.45 \mathrm{eV}$ peak is that it originates from the GaAs substrate. However, this peak was not observed in the PL spectrum of sample \#GT1, and the PL maximum is shifted by $\sim 50 \mathrm{meV}$ toward a longer wavelength compared to the peak from the GaAs substrate. It is also highly unlikely that this peak is due to radiation from the hexagonal h-GaTe. Although the weak broad PL emission at $1.44 \mathrm{eV}(\mathrm{T}=300 \mathrm{~K})$ was observed by Fonseca et al. [116] from the hexagonal $\mathrm{GaSe}_{\mathrm{x}} \mathrm{Te}_{1-\mathrm{x}}$ islands with low Se content $(\mathrm{x} \leq 0.05)$, according to Cai et al. [117], the hexagonal GaTe should possess the bandgap at $1.03 \mathrm{eV}$, which does not correlate with intense emission at $1.45 \mathrm{eV}$. Moreover, the X-ray powder diffraction pattern of sample \#GT2 (Figure 18a) indicates the dominance of monoclinic GaTe [108,110]. Cai et al. [118] argued that this peak is associated with the emission centers localized at the domain edges. The dominance of this emission band $(1.45 \mathrm{eV})$ in our PL spectrum, measured at much lower excitation power density in comparison with that used by Cai et al., also supports this attribution. The absence of this peak in the sample \#GT1 grown at $\mathrm{T}_{\mathrm{S}} \approx 450{ }^{\circ} \mathrm{C}$ can be associated with much worse structural perfection of \#GT1 layer compared to \#GT2. The peak at $1.25 \mathrm{eV}$, which is the only one registered in the PL spectra of both samples (\#GT1 and \#GT2), probably corresponds to the radiation of a gallium vacancy complex in the GaAs substrate [119]. This assumption was confirmed by observation of the same peak in the PL spectrum of the bare GaAs substrate without the GaTe layer. Nevertheless, this peak can also be related to the defect emission in GaTe, since the PL intensity at $1.25 \mathrm{eV}$ in sample \#GT2 is by an order of magnitude higher.

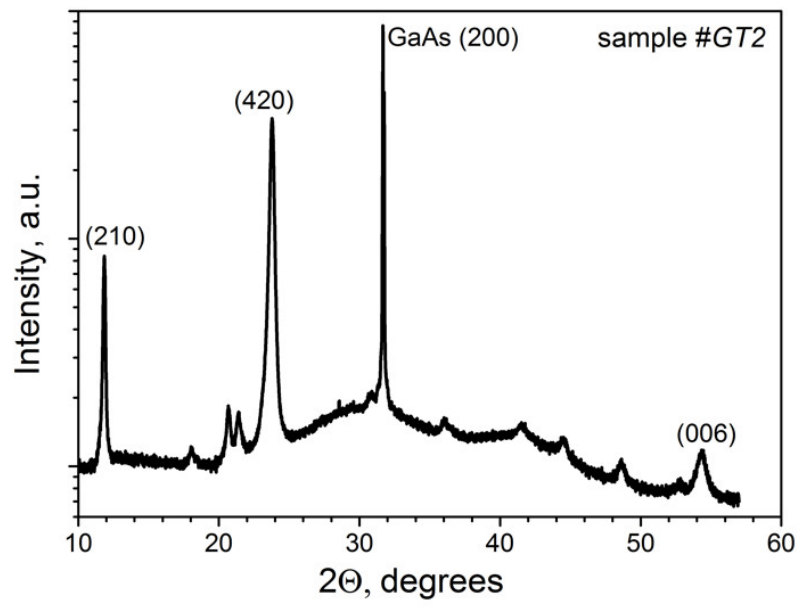

(a)

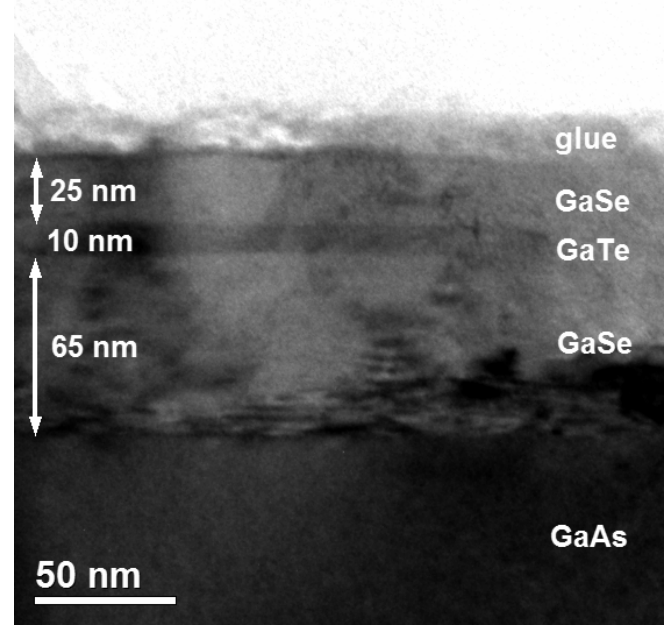

(b)

Figure 18. (a) The X-ray powder diffraction pattern of GaTe layer grown at $\mathrm{T}_{\mathrm{S}} \approx 530{ }^{\circ} \mathrm{C}$ (\#GT2); (b) cross-section TEM image of the GaSe/GaTe/GaSe heterostructure.

The GaSe/GaTe system is perspective for the fabrication of QW structures due to a type I band alignment at GaSe/GaTe interface [120]. The GaSe/GaTe/GaSe heterostructure was grown on GaAs(001) substrate with a 200-nm-thick GaAs buffer layer. To provide the relatively flat growth surface for the GaTe deposition, the bottom GaSe layer was grown at relatively low $\mathrm{T}_{\mathrm{S}}=400{ }^{\circ} \mathrm{C}$ with $\mathrm{Se} / \mathrm{Ga}(\mathrm{BEP}) \sim 20$ flux ratio. The average growth rate of GaSe was as low $\sim 1.3 \mathrm{~nm} / \mathrm{min}$. GaTe layer was deposited at $\mathrm{T}_{\mathrm{S}}=450{ }^{\circ} \mathrm{C}$, using the similar growth conditions as in sample \#GT1. The growth temperature of the top GaSe was chosen also equal to $\mathrm{T}_{\mathrm{S}}=400{ }^{\circ} \mathrm{C}$ to avoid the Se diffusion into the GaTe layers. The TEM image of the GaSe/GaTe/GaSe heterostructure is presented in Figure $18 \mathrm{~b}$. It can be seen that the GaTe layer differs in contrast from GaSe and forms a rather uniform layer. The interfaces 
between 2D materials are rather sharp. However, no PL has been revealed from the GaSe/GaTe/GaSe QW heterostructure. We assume that the main reason for the absence of PL in GaTe QW is a low growth temperature of both GaTe QW and GaSe barriers.

\section{Conclusions}

GaSe, InSe, and GaTe layers as well as the relevant heterostructures were grown by MBE on the GaAs(001) substrates at $T_{S}=400-530{ }^{\circ} \mathrm{C}$ using a standard Ga, In, and Te effusion cells as well as a Se valve cracking cell with the cracking zone temperature $\mathrm{T}_{\mathrm{Se}}(\mathrm{cr})=500^{\circ} \mathrm{C}$ as molecular beam sources. The structures were studied in detail using SEM, TEM, X-ray diffraction, Raman, and PL spectroscopy techniques. The growth conditions corresponding to the stoichiometric ratio of $\mathrm{Ga}$ and Se adatoms on the growth surface as a function of growth temperature, as well as the correlation between the growth conditions and the properties of the GaSe layers were established. Powder X-ray diffraction has shown that the GaSe layers grown under strong Se-rich conditions contain inclusions of the secondary $\mathrm{Ga}_{2} \mathrm{Se}_{3}$ phase. A combination of SAED and Raman spectroscopy techniques confirmed that the GaSe layers with a relatively flat surface morphology grown at $\mathrm{T}_{\mathrm{S}}=400{ }^{\circ} \mathrm{C}$ correspond to the $\gamma$-GaSe polytype, and the $c$ axis of the GaSe layer in this case is normal to the substrate/layer interface. Contrary to that, GaSe layers grown at high $\mathrm{T}_{\mathrm{S}}=500{ }^{\circ} \mathrm{C}$ on the $\mathrm{GaAs}(001)$ substrates predominantly possess an $\varepsilon$-GaSe polytype, and the TEM images indicate the preferential orientation of the $c$ axis of $\varepsilon$-GaSe along the $<111>$ directions of the GaAs substrate due to the chemical interaction between the substrate and the growing layer. Nevertheless, the formation of a particular GaSe-polytype is governed not only by the growth temperature, but rather by a combination of various factors. The RT near band-edge excitonic photoluminescence in the $\mathrm{GaSe} / \mathrm{GaAs}(001)$ layers grown at $\mathrm{T}_{\mathrm{S}} \sim 500{ }^{\circ} \mathrm{C}$ was demonstrated, while the intense emission band centered at $\sim 1.7 \mathrm{eV}$ dominates the PL spectra of the GaSe/GaAs(001) layers grown at low $\mathrm{T}_{\mathrm{S}} \sim 400{ }^{\circ} \mathrm{C}$ temperature.

The MBE growth of InSe directly on the $\mathrm{GaAs}(001)$ was found to be a challenge due to formation of numerous $\mathrm{In}_{\mathrm{x}} \mathrm{Se}_{\mathrm{y}}$-type phases in the InSe film. In the low-temperature ( $\mathrm{T}=11 \mathrm{~K}$ ) PL spectra of InSe/GaAs(001) layers, the highest-energy peak was detected at $\sim 1.307 \mathrm{eV}$. The temperature dependence of the PL spectrum revealed that this peak consists of the two closely-spaced and poorly separated bands, which were attributed to the recombination of native acceptor and donor-acceptor pairs, respectively. Taking account of difficulties in InSe/GaAs(001) heteroepitaxy, we tried to realize the $\mathrm{ZnSe} / \mathrm{InSe} / \mathrm{ZnSe}$ quantum well $(\mathrm{QW})$ heterostructures with the thickness of InSe layers varied within 2-7 TLs range. The TEM data reveal the island character of the InSe/ZnSe(001) nucleation; however, all QW structures demonstrated the strong intense broad PL band centered at $\sim 2.27 \mathrm{eV}$, regardless of the QW width. This PL band was associated with radiative recombination centers of In in ZnSe because of the In diffusion into the underlying ZnSe layer. In addition, the strong diffusion of Ga into the top InSe layer was observed in InSe/GaSe/GaAs(001) structures at InSe growth temperature of $\mathrm{T}_{\mathrm{S}} \sim 450{ }^{\circ} \mathrm{C}$.

The near band-edge PL at $\mathrm{T}=11 \mathrm{~K}$ was observed in GaTe layer grown at high $\mathrm{T}_{\mathrm{S}} \sim 530{ }^{\circ} \mathrm{C}$. The peak with energy of $\sim 1.72 \mathrm{eV}$ was associated with the emission of excitons bound at the acceptor in $\mathrm{GaTe}$. However, no excitonic PL was observed in the GaSe/GaTe/GaSe QW heterostructure, probably due to low growth temperature of both GaTe QW and GaSe barriers, suggesting the thesis that growth temperature is the main factor affecting the optical quality of the III-VI layers. Nevertheless, the GaSe/GaTe material system looks as the most prospective for the fabrication of the optically active QW structures due to the ability to grow the relatively flat GaTe layers at high growth temperature $\mathrm{T}_{\mathrm{S}}>500^{\circ} \mathrm{C}$.

Supplementary Materials: The following are available online at http://www.mdpi.com/1996-1944/13/16/3447/s1, Figure S1: The dependence of surface morphology of GaSe/GaAs(001) layers grown using standard Ga and Se valve cracking cell with $\mathrm{T}_{\mathrm{Se}}(\mathrm{cr})=500{ }^{\circ} \mathrm{C}$ on both $\mathrm{P}_{\mathrm{Se}} / \mathrm{P}_{\mathrm{Ga}}(\mathrm{BEP})$ ratio and substrate temperature. GaSe layers with and without $\mathrm{Ga}$ droplets on the growth surface are indicated by the hollow and filled circles, Figure S2: RHEED patterns during the growth of GaSe layers: (a) \#GS6, $\mathrm{T}_{\mathrm{S}} \sim 400{ }^{\circ} \mathrm{C}$, $\mathrm{Se} / \mathrm{Ga}(\mathrm{BEP})=12, \mathrm{r}_{\mathrm{GaSe}} \sim 1.5 \mathrm{~nm} / \mathrm{min}$; and $(\mathrm{b}) \# G S 7, \mathrm{~T}_{\mathrm{S}} \sim 500^{\circ} \mathrm{C}, \mathrm{Se} / \mathrm{Ga}(\mathrm{BEP})=25, \mathrm{r}_{\mathrm{GaSe}} \sim 2.1 \mathrm{~nm} / \mathrm{min}$, Figure S3: (a) The selective area electron diffraction pattern of GaSe layer grown on a $\mathrm{GaAs}(001)$ substrate at $\mathrm{T}_{\mathrm{S}} \sim 400{ }^{\circ} \mathrm{C}$ under strong Se-rich conditions (\#GS1); 
and (b) cross-section TEM image, demonstrating an existence of the $\mathrm{Ga}_{2} \mathrm{Se}_{3}$ phase inclusions in the same layer, Figure S4: Cross-section TEM (a) and HRTEM (b) images of the GaSe layer grown on GaAs(001) substrate at $\mathrm{T}_{\mathrm{S}} \sim 500{ }^{\circ} \mathrm{C}(\mathrm{Se} / \mathrm{Ga}(\mathrm{BEP}) \sim 25)$ (sample \#GS7), Figure S5: Raman spectrum of the bulk $\varepsilon$-GaSe layer produced by HQ Graphene-curve 1. Typical Raman spectra of $\gamma$-GaSe layers grown on GaAs(001) and GaAs(112) substrates at $\mathrm{T}_{\mathrm{S}} \sim 400{ }^{\circ} \mathrm{C}$ (Curves 2 and 3, respectively). All spectra are normalized to the intensity of the $\mathrm{A}_{1}\left(132 \mathrm{~cm}^{-1}\right)$ phonon line, Figure S6: Photoluminescence spectrum of the $\mathrm{InSe} / \mathrm{GaAs}(001)$ layer measured at $\mathrm{T}=11 \mathrm{~K}$. The spectrum is most accurately approximated by four Gaussian contours centered at 1.315, 1.301, 1.28, and $1.2 \mathrm{eV}$, respectively, Figure S7: The plan-view SEM image of the InSe/GaSe/GaAs(001) structure. The growth temperature of InSe layer was as high as $\mathrm{T}_{\mathrm{S}} \sim 450{ }^{\circ} \mathrm{C}$, Figure S8: PL spectra of the GaTe/GaAs(001) layers \#GT1 and \#GT2 measured at the same excitation power density of $0.5 \mathrm{~W} / \mathrm{cm}^{2}$.

Author Contributions: Conceptualization, S.V.S.; Funding acquisition, S.V.I. and D.D.F.; Investigation, S.V.S., P.S.A., I.V.S., D.A.K., V.Y.D., O.S.K., and D.D.F.; Methodology, S.V.S., P.S.A., and V.Y.D.; Project administration, S.V.S. and S.V.I.; Supervision, S.V.S. and S.V.I.; Visualization, S.V.S., P.S.A., D.A.K., V.Y.D., O.S.K., and D.D.F.; Writing—original draft, S.V.S.; and Writing—review and editing, P.S.A., I.V.S., V.Y.D., O.S.K., D.D.F., and S.V.I. All authors have read and agreed to the published version of the manuscript.

Funding: The authors from St. Petersburg Electrotechnical University (O.S. Komkov, D.D. Firsov) acknowledge the support of the Russian Science Foundation (RSF), project \#18-79-10161. TEM and XRD characterization were performed in the Joint Research Center "Material science and characterization in advanced technology" with partial financial support from the Ministry of Education and Science of the Russian Federation.

Acknowledgments: The authors are thankful to S. Troshkov, M. Yagovkina and A. Smirnov for the SEM, XRD, and Raman measurements. The authors also note the contribution of D. Kolyada from St. Petersburg Electrotechnical University as well as M. Rakhlin and A. Galimov from Ioffe Institute to the PL measurements.

Conflicts of Interest: The authors declare no conflict of interest. The funders had no role in the design of the study; in the collection, analyses, or interpretation of data; in the writing of the manuscript, or in the decision to publish the results.

\section{References}

1. Huang, W.; Gan, L.; Li, H.; Ma, Y.; Zhai, T. 2D layered group IIIA metal chalcogenides: Synthesis, properties and applications in electronics and optoelectronics. CrystEngComm 2016, 18, 3968-3984. [CrossRef]

2. Xu, K.; Yin, L.; Huang, Y.; Shifa, T.A.; Chu, J.; Wang, F.; Cheng, R.; Wang, Z.; He, J. Synthesis, properties and applications of 2D layered MIIIXVI(M = Ga, In; X = S, Se, Te) materials. Nanoscale 2016, 8, 16802-16818. [CrossRef] [PubMed]

3. Bandurin, D.A.; Tyurnina, A.V.; Yu, G.L.; Mishchenko, A.; Zólyomi, V.; Morozov, S.V.; Kumar, R.K.; Gorbachev, R.V.; Kudrynskyi, Z.R.; Pezzini, S.; et al. High electron mobility, quantum Hall effect and anomalous optical response in atomically thin InSe. Nat. Nanotechnol. 2016, 12, 223-227. [CrossRef] [PubMed]

4. Late, D.J.; Liu, B.; Luo, J.; Yan, A.; Matte, H.S.S.R.; Grayson, M.; Rao, C.N.R.; Dravid, V.P. GaS and GaSe Ultrathin Layer Transistors. Adv. Mater. 2012, 24, 3549-3554. [CrossRef]

5. Liu, X.; Ren, J.-C.; Shen, T.; Li, S.; Liu, W. Lateral InSe p-n Junction Formed by Partial Doping for Use in Ultrathin Flexible Solar Cells. J. Phys. Chem. Lett. 2019, 10, 7712-7718. [CrossRef]

6. Chen, Z.; Chu, S.; Chen, J.; Chen, H.; Zhang, J.; Ma, X.; Li, Q.; Chen, X. Epitaxially grown semi-vertical and aligned GaTe two dimensional sheets on $\mathrm{ZnO}$ substrate for energy harvesting applications. Nano Energy 2019, 56, 294-299. [CrossRef]

7. Wang, F.; Wang, Z.; Yin, L.; Cheng, R.; Wang, J.; Wen, Y.; Shifa, T.A.; Wang, F.; Zhang, Y.; Zhan, X.; et al. 2D library beyond graphene and transition metal dichalcogenides: A focus on photodetection. Chem. Soc. Rev. 2018, 47, 6296-6341. [CrossRef]

8. Yang, Z.; Hao, J. Recent Progress in 2D Layered III-VI Semiconductors and their Heterostructures for Optoelectronic Device Applications. Adv. Mater. Technol. 2019, 4, 1900108. [CrossRef]

9. Yao, J.; Zheng, Z.; Yang, G. Production of large-area 2D materials for high-performance photodetectors by pulsed-laser deposition. Prog. Mater. Sci. 2019, 106, 100573. [CrossRef]

10. Tanabe, T.; Suto, K.; Nishizawa, J.-I.; Sasaki, T. Characteristics of terahertz-wave generation from GaSe crystals. J. Phys. D Appl. Phys. 2003, 37, 155-158. [CrossRef]

11. Dong, J.; Gradwohl, K.-P.; Xu, Y.; Wang, T.; Zhang, B.-B.; Xiao, B.; Teichert, C.; Jie, W. Terahertz emission from layered GaTe crystal due to surface lattice reorganization and in-plane noncubic mobility anisotropy. Photon. Res. 2019, 7, 518-525. [CrossRef] 
12. Ben Aziza, Z.; Pierucci, D.; Henck, H.; Silly, M.G.; David, C.; Yoon, M.; Sirotti, F.; Xiao, K.; Eddrief, M.; Girard, J.-C.; et al. Tunable quasiparticle band gap in few-layer GaSe/graphene van der Waals heterostructures. Phys. Rev. B 2017, 96, 035407. [CrossRef]

13. Mudd, G.W.; Molas, M.R.; Chen, X.; Zólyomi, V.; Nogajewski, K.; Kudrynskyi, Z.R.; Kovalyuk, Z.D.; Yusa, G.; Makarovsky, O.; Eaves, L.; et al. The direct-to-indirect band gap crossover in two-dimensional van der Waals Indium Selenide crystals. Sci. Rep. 2016, 6, 39619. [CrossRef] [PubMed]

14. Terry, D.J.; Zolyomi, V.; Hamer, M.J.; Tyurnina, A.V.; Hopkinson, D.; Rakowski, A.M.; Magorrian, S.; Clark, N.; Andreev, Y.M.; Kazakova, O.; et al. Infrared-to-violet tunable optical activity in atomic films of GaSe, InSe, and their heterostructures. 2D Mater. 2018, 5, 041009. [CrossRef]

15. Cai, H.; Gu, Y.; Lin, Y.-C.; Yu, Y.; Geohegan, D.B.; Xiao, K. Synthesis and emerging properties of 2D layered III-VI metal chalcogenides. Appl. Phys. Rev. 2019, 6, 041312. [CrossRef]

16. Geim, A.K.; Grigorieva, I.V. Van der Waals heterostructures. Nature 2013, 499, 419-425. [CrossRef]

17. Koma, A. Van der Waals epitaxy for highly lattice-mismatched systems. J. Cryst. Growth 1999, $201,236-241$. [CrossRef]

18. Yuan, X.; Tang, L.; Wang, P.; Chen, Z.-G.; Zou, Y.-C.; Su, X.; Zhang, C.; Liu, Y.; Wang, W.; Liu, C.; et al. Wafer-scale arrayed p-n junctions based on few-layer epitaxial GaTe. Nano Res. 2015, 8, 3332-3341. [CrossRef]

19. Yuan, X.; Tang, L.; Liu, S.; Wang, P.; Chen, Z.-G.; Zhang, C.; Liu, Y.; Wang, W.; Zou, Y.; Liu, C.; et al. Arrayed van der Waals Vertical Heterostructures Based on 2D GaSe Grown by Molecular Beam Epitaxy. Nano Lett. 2015, 15, 3571-3577. [CrossRef]

20. Liu, S.; Yuan, X.; Wang, P.; Chen, Z.-G.; Tang, L.; Zhang, E.; Zhang, C.; Liu, Y.; Wang, W.; Liu, C.; et al. Controllable Growth of Vertical Heterostructure GaTexSe1-x/Si by Molecular Beam Epitaxy. ACS Nano 2015, 9, 8592-8598. [CrossRef]

21. Ueno, K.; Abe, H.; Saiki, K.; Koma, A. Heteroepitaxy of Layered Semiconductor GaSe on a GaAs(111)B Surface. Jpn. J. Appl. Phys. 1991, 30, L1352-L1354. [CrossRef]

22. Emery, J.-Y.; Brahim-Ostmane, L.; Hirlimann, C.; Chevy, A. Reflection high-energy electron diffraction studies of InSe and GaSe layered compounds grown by molecular beam epitaxy. J. Appl. Phys. 1992, 71, 3256-3259. [CrossRef]

23. Ghalouci, L.; Benbahi, B.; Hiadsi, S.; Abidri, B.; Vergoten, G.; Ghalouci, F. First principle investigation into hexagonal and cubic structures of Gallium Selenide. Comput. Mater. Sci. 2013, 67, 73-82. [CrossRef]

24. Brudnyi, V.N.; Kosobutsky, A.V.; Sarkisov, S.Y. Charge neutrality level and electronic properties of GaSe under pressure. Semiconductors 2010, 44, 1158-1166. [CrossRef]

25. Allakhverdiev, K.R.; Yetis, M.Ö.; Özbek, S.; Baykara, T.K.; Salaev, E.Y. Effective nonlinear GaSe crystal. Optical properties and applications. Laser Phys. 2009, 19, 1092-1104. [CrossRef]

26. Kuhn, A.; Chevy, A.; Chevalier, R. Crystal structure and interatomic distances in GaSe. Phys. Status Solidi (A) 1975, 31, 469-475. [CrossRef]

27. De Blasi, C.; Manno, D.; Rizzo, A. Convergent-beam electron diffraction study of melt-and vapour-grown single crystals of gallium chalcogenides. Il Nuovo Cimento D 1989, 11, 1145-1163. [CrossRef]

28. Sorokin, S.V.; Avdienko, P.S.; Sedova, I.V.; Kirilenko, D.A.; Yagovkina, M.A.; Smirnov, A.N.; Davydov, V.Y.; Ivanov, S.V. Molecular-Beam Epitaxy of Two-Dimensional GaSe Layers on GaAs(001) and GaAs(112) Substrates: Structural and Optical Properties. Semiconductors 2019, 53, 1131-1137. [CrossRef]

29. Dai, Z.; Chegwidden, S.R.; Rumaner, L.E.; Ohuchi, F.S. Microstructure evolution of GaSe thin films grown on GaAs(100) by molecular beam epitaxy. J. Appl. Phys. 1999, 85, 2603-2608. [CrossRef]

30. Lee, C.H.; Krishnamoorthy, S.; O’Hara, D.; Brenner, M.R.; Johnson, J.M.; Jamison, J.S.; Myers, R.C.; Kawakami, R.K.; Hwang, J.; Rajan, S. Molecular beam epitaxy of 2D-layered gallium selenide on GaN substrates. J. Appl. Phys. 2017, 121, 094302. [CrossRef]

31. Ueno, K.; Takeda, N.; Sasaki, K.; Koma, A. Investigation of the growth mechanism of layered semiconductor GaSe. Appl. Surf. Sci. 1997, 113, 38-42. [CrossRef]

32. Beechem, T.E.; Kowalski, B.M.; Brumbach, M.T.; McDonald, A.E.; Spataru, C.D.; Howell, S.W.; Ohta, T.; Pask, J.A.; Kalugin, N.G. Oxidation of ultrathin GaSe. Appl. Phys. Lett. 2015, 107, 173103. [CrossRef]

33. Susoma, J.; Lahtinen, J.; Kim, M.; Riikonen, J.; Lipsanen, H. Crystal quality of two-dimensional gallium telluride and gallium selenide using Raman fingerprint. AIP Adv. 2017, 7, 015014. [CrossRef]

34. Rahaman, M.; Rodriguez, R.D.; Monecke, M.; Lopez-Rivera, S.A.; Zahn, D.R.T. GaSe oxidation in air: From bulk to monolayers. Semicond. Sci. Technol. 2017, 32, 105004. [CrossRef] 
35. Drapak, S.; Gavrylyuk, S.V.; Kovalyuk, Z.D.; Lytvyn, O. Native oxide emerging of the cleavage surface of gallium selenide due to prolonged storage. Semiconductors 2008, 42, 414-421. [CrossRef]

36. Han, G.; Chen, Z.-G.; Drennan, J.; Zou, J. Indium Selenides: Structural Characteristics, Synthesis and Their Thermoelectric Performances. Small 2014, 10, 2747-2765. [CrossRef]

37. Camassel, J.; Merle, P.; Mathieu, H.; Chevy, A. Excitonic absorption edge of indium selenide. Phys. Rev. B 1978, 17, 4718-4725. [CrossRef]

38. Politano, A.; Campi, D.; Cattelan, M.; Ben Amara, I.; Jaziri, S.; Mazzotti, A.; Barinov, A.; Gürbulak, B.; Duman, S.; Agnoli, S.; et al. Indium selenide: An insight into electronic band structure and surface excitations. Sci. Rep. 2017, 7, 3445. [CrossRef]

39. Hao, Q.; Yi, H.; Su, H.; Wei, B.; Wang, Z.; Lao, Z.; Chai, Y.; Wang, Z.; Jin, C.; Dai, J.; et al. Phase Identification and Strong Second Harmonic Generation in Pure $\varepsilon$-InSe and Its Alloys. Nano Lett. 2019, 19, 2634-2640. [CrossRef]

40. Popović, S.; Tonejc, A.; Grzeta-Plenkovic, B.; Čelustka, B.; Trojko, R. Revised and new crystal data for indium selenides. J. Appl. Crystallogr. 1979, 12, 416-420. [CrossRef]

41. Chevy, A.; Kuhn, A.; Martin, M.-S. Large InSe monocrystals grown from a non-stoichiometric melt. J. Cryst. Growth 1977, 38, 118-122. [CrossRef]

42. Nagpal, K.C.; Ali, S.Z. An X-ray study of InSe. Indian J. Pure Appl. Phys. 1975, 13, 258-264.

43. Lei, S.; Ge, L.; Najmaei, S.; George, A.; Kappera, R.; Lou, J.; Chhowalla, M.; Yamaguchi, H.; Gupta, G.; Vajtai, R.; et al. Evolution of the Electronic Band Structure and Efficient Photo-Detection in Atomic Layers of InSe. ACS Nano 2014, 8, 1263-1272. [CrossRef] [PubMed]

44. Huang, S.; Tatsumi, Y.; Ling, X.; Guo, H.; Wang, Z.; Watson, G.; Puretzky, A.A.; Geohegan, D.B.; Kong, J.; Li, J.; et al. In-Plane Optical Anisotropy of Layered Gallium Telluride. ACS Nano 2016, 10, 8964-8972. [CrossRef] [PubMed]

45. Wang, Z.; Xu, K.; Li, Y.; Zhan, X.; Safdar, M.; Wang, Q.; Wang, F.; He, J. Role of Ga Vacancy on a Multilayer GaTe Phototransistor. ACS Nano 2014, 8, 4859-4865. [CrossRef] [PubMed]

46. Kolesnikov, N.; Borisenko, E.; Borisenko, D.; Timonina, A. Structure and microstructure of GaTe crystals grown by High-Pressure vertical zone melting. J. Cryst. Growth 2013, 365, 59-63. [CrossRef]

47. Fonseca, J.J.; Tongay, S.; Topsakal, M.; Chew, A.R.; Lin, A.J.; Ko, C.; Luce, A.V.; Salleo, A.; Wu, J.; Dubon, O. Bandgap Restructuring of the Layered Semiconductor Gallium Telluride in Air. Adv. Mater. 2016, 28, 6465-6470. [CrossRef]

48. Komkov, O.S.; Firsov, D.D.; Chernov, M.Y.; Solov'Ev, V.A.; Sitnikova, A.A.; Kop'Ev, P.S.; Ivanov, S.V. Radiative versus non-radiative recombination in high-efficiency mid-IR $\operatorname{InSb} / \operatorname{InAs} / \operatorname{In}(\mathrm{Ga}, \mathrm{Al}) \mathrm{As} / \mathrm{GaAs}$ metamorphic nanoheterostructures. J. Phys. D Appl. Phys. 2018, 51, 055106. [CrossRef]

49. Kojima, N.; Sato, K.; Yamada, A.; Konagai, M.; Takahashi, K. Epitaxial Growth of GaSe Films by Molecular Beam Epitaxy on GaAs(111), (001) and (112) Substrates. Jpn. J. Appl. Phys. 1994, 33, L1482-L1484. [CrossRef]

50. Kojima, N.; Sato, K.; Budiman, M.; Yamada, A.; Konagai, M.; Takahashi, K.; Nakamura, Y.; Nittono, O. Molecular beam epitaxial growth and characterization of epitaxial GaSe films on (001)GaAs. J. Cryst. Growth 1995, 150, 1175-1179. [CrossRef]

51. Budiman, M.; Okamoto, T.; Yamada, A.; Konagai, M. Epitaxial growth and characterization of GaSxSe1-x layered compound semiconductor by molecular beam epitaxy. Appl. Surf. Sci. 1997, 117, 518-522. [CrossRef]

52. Rumaner, L.E.; Gray, J.; Ohuchi, F. Nucleation and growth of GaSe on GaAs by Van der Waal epitaxy. J. Cryst. Growth 1997, 177, 17-27. [CrossRef]

53. Rumaner, L.E. Interaction of GaSe with GaAs(111): Formation of heterostructures with large lattice mismatch. J. Vac. Sci. Technol. B Microelectron. Nanometer Struct. 1998, 16, 977. [CrossRef]

54. Novoselov, K.; Geim, A.K.; Morozov, S.; Jiang, D.; Zhang, Y.; Dubonos, S.V.; Grigorieva, I.V.; Firsov, A.A. Electric Field Effect in Atomically Thin Carbon Films. Science 2004, 306, 666-669. [CrossRef] [PubMed]

55. Diep, N.Q.; Liu, C.-W.; Wu, S.-K.; Chou, W.-C.; Huynh, S.H.; Chang, E.Y. Screw-Dislocation-Driven Growth Mode in Two Dimensional GaSe on GaAs(001) Substrates Grown by Molecular Beam Epitaxy. Sci. Rep. 2019, 9, 1-8. [CrossRef] [PubMed]

56. Ueno, K.; Sakurai, M.; Koma, A. Van der Waals epitaxy on hydrogen-terminated Si(111) surfaces and investigation of its growth mechanism by atomic force microscope. J. Cryst. Growth 1995, 150, 1180-1185. [CrossRef] 
57. Nie, Y.; Barton, A.T.; Addou, R.; Zheng, Y.; Walsh, L.; Eichfeld, S.M.; Yue, R.; Cormier, C.R.; Zhang, C.; Wang, Q.; et al. Dislocation driven spiral and non-spiral growth in layered chalcogenides. Nanoscale 2018, 10, 15023-15034. [CrossRef]

58. Chen, M.; Kim, H.; Ovchinnikov, D.; Kuc, A.B.; Heine, T.; Renault, O.; Kis, A. Large-grain MBE-grown GaSe on GaAs with a Mexican hat-like valence band dispersion. npj 2D Mater. Appl. 2018, 2, 2. [CrossRef]

59. Ivanov, S.; Sorokin, S.; Sedova, I.V. Molecular Beam Epitaxy of Wide Gap II-VI Laser Heterostructures; Elsevier BV: Oxford, UK, 2018; Chapter 25; pp. 571-595.

60. Komkov, O.S.; Khakhulin, S.A.; Firsov, D.D.; Avdienko, P.S.; Sedova, I.V.; Sorokin, S.V. Investigation of built-in electric fields at the GaSe/GaAs interface by photoreflectance spectroscopy. Semiconductors 2020, 54, 1011-1017.

61. Dai, Z.; Ohuchi, F.S. Vacancy ordering of Ga2Se3 at GaSe/GaAs(100) interface. Appl. Phys. Lett. 1998, 73, 966-968. [CrossRef]

62. Afifi, M.; Bekheet, A.; El-Shair, H.; Zedan, I. Determination and analysis of optical constants for Ga2Se3 films near absorption edge. Phys. B Condens. Matter 2003, 325, 308-318. [CrossRef]

63. Irwin, J.; Hoff, R.; Clayman, B.; Bromley, R. Long wavelength lattice vibrations in GaS and GaSe. Solid State Commun. 1973, 13, 1531-1536. [CrossRef]

64. Hoff, R.M.; Irwin, J.C.; Lieth, R.M.A. Raman Scattering in GaSe. Can. J. Phys. 1975, 53, 1606-1614. [CrossRef]

65. Lim, S.Y.; Lee, J.-U.; Kim, J.H.; Liang, L.; Kong, X.; Nguyen, T.T.H.; Lee, Z.; Cho, S.; Cheong, H. Polytypism in few-layer gallium selenide. Nanoscale 2020, 12, 8563-8573. [CrossRef]

66. Yoshida, H.; Nakashima, S.; Mitsuishi, A. Phonon Raman spectra of layer compound GaSe. Phys. Status Solidi (B) 1973, 59, 655-666. [CrossRef]

67. Bergeron, A.; Ibrahim, J.; Leonelli, R.; Francoeur, S. Oxidation dynamics of ultrathin GaSe probed through Raman spectroscopy. Appl. Phys. Lett. 2017, 110, 241901. [CrossRef]

68. Longuinhos, R.; Ribeiro-Soares, J.; Longuinhos, R. Ultra-weak interlayer coupling in two-dimensional gallium selenide. Phys. Chem. Chem. Phys. 2016, 18, 25401-25408. [CrossRef]

69. Polian, A.; Kunc, K.; Kuhn, A. Low-frequency lattice vibrations of $\delta$-GaSe compared to $\epsilon$ - and $\gamma$-polytypes. Solid State Commun. 1976, 19, 1079-1082. [CrossRef]

70. Zhou, J.; Wu, Y.; Wang, H.; Wu, Z.; Li, X.; Yang, W.; Ke, C.; Lu, S.; Zhang, C.; Kang, J.; et al. Strain manipulation of the polarized optical response in two-dimensional GaSe layers. Nanoscale 2020, 12, 4069-4076. [CrossRef]

71. Cai, H.; Soignard, E.; Ataca, C.; Chen, B.; Ko, C.; Aoki, T.; Pant, A.; Meng, X.; Yang, S.; Grossman, J.; et al. Band Engineering by Controlling vdW Epitaxy Growth Mode in 2D Gallium Chalcogenides. Adv. Mater. 2016, 28, 7375-7382. [CrossRef]

72. Capozzi, V.; Montagna, M. Optical spectroscopy of extrinsic recombinations in gallium selenide. Phys. Rev. B 1989, 40, 3182-3190. [CrossRef] [PubMed]

73. Budiman, M.; Yamada, A.; Konagai, M. Heteroepitaxy of Layered Compound InSe and InSe/GaSe onto GaAs Substrates. Jpn. J. Appl. Phys. 1998, 37, 4092-4098. [CrossRef]

74. Capozzi, V. Direct and indirect excitonic emission in GaSe. Phys. Rev. B 1981, 23, 836-840. [CrossRef]

75. Avdienko, P.; Sorokin, S.; Sedova, I.; Kirilenko, D.; Smirnov, A.; Eliseev, I.; Davydov, V.; Ivanov, S. Structural and Optical Properties of GaSe/GaAs(001) Layers Grown by Molecular Beam Epitaxy. Acta Phys. Pol. A 2019, 136, 608-612. [CrossRef]

76. Chatillon, C. Critical analysis of the thermodynamic properties of the In-Se gaseous and solid phases. J. Cryst. Growth 1993, 129, 297-311. [CrossRef]

77. Emery, J.; Julien, C.; Jouanne, M.; Balkanski, M. Growth conditions and structure of polycrystalline InSe thin films. Appl. Surf. Sci. 1988, 33, 619-624. [CrossRef]

78. Emery, J.; Brahim-Otsmane, L.; Jouanne, M.; Julien, C.; Balkanski, M. Growth conditions of films by molecular beam deposition. Mater. Sci. Eng. B 1989, 3, 13-17. [CrossRef]

79. Brahim-Otsmane, L.; Emery, J.-Y.; Eddrief, M. X-ray, reflection high electron energy diffraction and X-ray photoelectron spectroscopy studies of InSe and $\gamma$-In2Se3 thin films grown by molecular beam deposition. Thin Solid Films 1994, 237, 291-296. [CrossRef]

80. Jasinski, J.; Liliental-Weber, Z.; Chaiken, A.; Gibson, G.; Nauka, K.; Yang, C.; Bicknell, R. Heteroepitaxy of InSe/GaSe on Si(111) Substrates. MRS Symp. Proc. 2003, 803. [CrossRef]

81. Chatillon, C.; Emery, J.-Y. Thermodynamic analysis of molecular beam epitaxy of compounds in the In-Se system. J. Cryst. Growth 1993, 129, 312-320. [CrossRef] 
82. Ivanov, S.; Sorokin, S.; Kop'Ev, P.; Kim, J.; Jung, H.; Park, H. Composition, stoichiometry and growth rate control in molecular beam epitaxy of ZnSe based ternary and quaternary alloys. J. Cryst. Growth 1996, 159, 16-20. [CrossRef]

83. Ivanov, S.; Sorokin, S.; Krestnikov, I.; Faleev, N.; Ber, B.; Sedova, I.; Kudryavtsev, Y.; Kop'Ev, P. Interplay of kinetics and thermodynamics in molecular beam epitaxy of (Mg,Zn,Cd)(S,Se). J. Cryst. Growth 1998, 184, 70-74. [CrossRef]

84. Julien, C.; Khelfa, A.; Benramdane, N.; Guesdon, J. Preparation and characterization of In4Se3 films. Mater. Sci. Eng. B 1994, 27, 53-60. [CrossRef]

85. Zhai, Y.; Zhang, Q.; Jiang, J.; Zhang, T.; Xiao, Y.; Yang, S.; Xu, G. Thermoelectric performance of the ordered In4Se3-In composite constructed by monotectic solidification. J. Mater. Chem. A 2013, 1, 8844. [CrossRef]

86. Milutinović, A.; Lazarević, Z.Ž.; Jakovljević, M.; Hadzić, B.; Petrović, M.; Gilić, M.; Dobrowolski, W.D.; Romčević, N.Ž. Optical properties of layered III-VI semiconductor $\gamma$-InSe: M ( M = Mn, Fe, Co, Ni). J. Phys. Chem. Solids 2016, 89, 120-127. [CrossRef]

87. Carlone, C.; Jandl, S.; Shanks, H.R. Optical Phonons and Crystalline Symmetry of InSe. Phys. Status Solidi (B) 1981, 103, 123-130. [CrossRef]

88. Segura, A. Layered Indium Selenide under High Pressure: A Review. Crystals 2018, 8, 206. [CrossRef]

89. Wu, M.; Xie, Q.; Wu, Y.; Zheng, J.; Wang, W.; He, L.; Wu, X.; Lv, B. Crystal structure and optical performance in bulk $\gamma$-InSe single crystals. AIP Adv. 2019, 9, 025013. [CrossRef]

90. Grimaldi, I.; Gerace, T.; Pipita, M.; Perrotta, I.; Ciuchi, F.; Berger, H.; Papagno, M.; Castriota, M.; Pacilé, D. Structural investigation of InSe layered semiconductors. Solid State Commun. 2020, 311, 113855. [CrossRef]

91. Kuroda, N.; Nishina, Y. Resonant Raman scattering at higher M0 exciton edge in layer compound InSe. Solid State Commun. 1978, 28, 439-443. [CrossRef]

92. Otsmane, L.B.; Emery, J.; Jouanne, M.; Balkanski, M. Epitaxy of layered semiconductor thin films. Appl. Surf. Sci. 1993, 65, 479-481. [CrossRef]

93. Hayashi, T.; Ueno, K.; Saiki, K.; Koma, A. Investigation of the growth mechanism of an InSe epitaxial layer on a MoS2 substrate. J. Cryst. Growth 2000, 219, 115-122. [CrossRef]

94. Shubina, T.V.; Desrat, W.; Moret, M.; Tiberj, A.; Briot, O.; Davydov, V.Y.; Platonov, A.; Semina, M.A.; Gil, B. InSe as a case between 3D and 2D layered crystals for excitons. Nat. Commun. 2019, 10, 3479. [CrossRef] [PubMed]

95. Abha Photoluminescence studies on the layer semiconductor InSe. J. Appl. Phys. 1982, 53, 5169. [CrossRef]

96. Abay, B.; Efeoğlu, H.; Yoğurtçu, Y. Low-temperature photoluminescence of n-InSe layer semiconductor crystals. Mater. Res. Bull. 1998, 33, 1401-1410. [CrossRef]

97. Homs, A.A.; Marí, B. Photoluminescence of undoped and neutron-transmutation-doped InSe. J. Appl. Phys. 2000, 88, 4654. [CrossRef]

98. Lang, O.; Klein, A.; Schlaf, R.; Löher, T.; Pettenkofer, C.; Jaegermann, W.; Chevy, A. heterointerfaces prepared by Van der Waals epitaxy. J. Cryst. Growth 1995, 146, 439-443. [CrossRef]

99. Royo, J.F.S.; Segura, A.; Lang, O.; Pettenkofer, C.; Jaegermann, W.; Chevy, A.; Roa, L. Photovoltaic properties of indium selenide thin films prepared by van der Waals epitaxy. Thin Solid Films 1997, 307, $283-287$. [CrossRef]

100. Wisotzki, E.; Klein, A.; Jaegermann, W. Quasi van der Waals epitaxy of ZnSe on the layered chalcogenides InSe and GaSe. Thin Solid Films 2000, 380, 263-265. [CrossRef]

101. Avdienko, P.S.; Kirilenko, D.A.; Sedova, I.V.; Sorokin, S.V. Structural properties of ZnSe/InSe/ZnSe heterostructures grown by molecular beam epitaxy on GaAs(001) substrates. J. Physics Conf. Ser. 2020, 1482, 012006. [CrossRef]

102. Smathers, J.B.; Kneedler, E.; Bennett, B.; Jonker, B. Nanometer scale surface clustering on ZnSe epilayers. Appl. Phys. Lett. 1998, 72, 1238-1240. [CrossRef]

103. Qidwai, A.; Woods, J. Defect levels in indium and gallium doped zinc selenide. J. Cryst. Growth 1982, 59, 217-222. [CrossRef]

104. Isshiki, M.; Yoshida, T.; Igaki, K.; Hayashi, Y.; Nakano, Y. Photoluminescence spectra of In-doped ZnSe single crystals. J. Phys. C Solid State Phys. 1986, 19, 4375-4381. [CrossRef]

105. Antonopoulos, J.G.; Karakostas, T.; Bleris, G.L.; Economou, N.A. On the phase diagram of the Ga-Te system in the composition range 55 at \% Te. J. Mater. Sci. 1981, 16, 733-738. [CrossRef] 
106. Teraguchi, N.; Kato, F.; Konagai, M.; Takahashi, K. Growth of III-VI Compound Semiconductors by Metalorganic Molecular Beam Epitaxy. Jpn. J. Appl. Phys. 1989, 28, L2134-L2136. [CrossRef]

107. Teraguchi, N.; Kato, F.; Konagai, M.; Takahashi, K. Growth and characterization of Ga2Te3 films by metalorganic molecular beam epitaxy. J. Electron. Mater. 1991, 20, 247-250. [CrossRef]

108. Bae, C.J.; McMahon, J.; Detz, H.; Strasser, G.; Park, J.; Einarsson, E.; Eason, D. Influence of thickness on crystallinity in wafer-scale GaTe nanolayers grown by molecular beam epitaxy. AIP Adv. 2017, 7, 35113. [CrossRef]

109. Zhao, Q.; Wang, T.; Miao, Y.; Ma, F.; Xie, Y.; Ma, X.; Gu, Y.; He, J.; Chen, B.; Xi, S.; et al. Thickness-induced structural phase transformation of layered gallium telluride. Phys. Chem. Chem. Phys. 2016, 18, 18719-18726. [CrossRef]

110. Borisenko, E.; Borisenko, D.; Timonina, A.; Kolesnikov, N. Nonvariant polymorphic transition from hexagonal to monoclinic lattice in GaTe single crystal. J. Cryst. Growth 2020, 535, 125548. [CrossRef]

111. Liu, F.; Shimotani, H.; Shang, H.; Kanagasekaran, T.; Zólyomi, V.; Drummond, N.D.; Fal’Ko, V.I.; Tanigaki, K. High-Sensitivity Photodetectors Based on Multilayer GaTe Flakes. ACS Nano 2014, 8, 752-760. [CrossRef]

112. Yu, Y.; Ran, M.; Zhou, S.; Wang, R.; Zhou, F.; Li, H.; Gan, L.; Zhu, M.; Zhai, T. Phase-Engineered Synthesis of Ultrathin Hexagonal and Monoclinic GaTe Flakes and Phase Transition Study. Adv. Funct. Mater. 2019. [CrossRef]

113. Gillan, E.G.; Barron, A.R. Chemical Vapor Deposition of Hexagonal Gallium Selenide and Telluride Films from Cubane Precursors: Understanding the Envelope of Molecular Control. Chem. Mater. 1997, 9, 3037-3048. [CrossRef]

114. Güder, H.; Abay, B.; Efeoğlu, H.; Yoğurtçu, Y.; Efeoğlu, H.; Yoğurtçu, Y. Photoluminescence characterization of GaTe single crystals. J. Lumin. 2001, 93, 243-248. [CrossRef]

115. Zubiaga, A.; García, J.A.; Plazaola, F.; Muñoz-Sanjose, V.; Martínez-Tomás, M.C. Recombination processes in unintentionally doped GaTe single crystals. J. Appl. Phys. 2002, 92, 7330-7336. [CrossRef]

116. Fonseca, J.J.; Horton, M.K.; Tom, K.; Yao, J.; Walukiewicz, W.; Dubón, O.D. Structure-Property Relationship of Low-Dimensional Layered GaSexTe1-x Alloys. Chem. Mater. 2018, 30, 4226-4232. [CrossRef]

117. Cai, H.; Chen, B.; Blei, M.; Chang, L.Y.; Wu, K.; Zhuang, H.; Tongay, S. Abnormal band bowing effects in phase instability crossover region of GaSe1-xTe x nanomaterials. Nat. Commun. 2018, 9, 1927. [CrossRef]

118. Cai, H.; Chen, B.; Wang, G.; Soignard, E.; Khosravi, A.; Manca, M.; Marie, X.; Chang, S.L.Y.; Urbaszek, B.; Tongay, S. Synthesis of Highly Anisotropic Semiconducting GaTe Nanomaterials and Emerging Properties Enabled by Epitaxy. Adv. Mater. 2016, 29, 1605551. [CrossRef]

119. Williams, E.W.; Bebb, H.B. Photoluminescence II: Gallium Arsenide. In Semiconductors and Semimetals; Willardson, R.K., Beer, A.C., Eds.; Academic Press: New York, NY, USA, 1972; Volume 8, Chapter 5; pp. 321-392.

120. Cai, H.; Kang, J.; Sahin, H.; Chen, B.; Suslu, A.; Wu, K.; Peeters, F.; Meng, X.; Tongay, S. Exciton pumping across type-I gallium chalcogenide heterojunctions. Nanotechnology 2016, 27, 065203. [CrossRef] 